\title{
Fricativas y africadas en lenguas campa entre los siglos X VIIIXX. Clasificación y diacronía de la familia campa
}

\author{
Carlos Alberto Faucet Pareja
}

Faucet Pareja, C. A. (2021). Fricativas y africadas en lenguas campa entre los siglos XVIIIXX.

Clasificación y diacronía de la familia campa. Revista de Filología y Lingüística de la Universidad de Costa Rica, 47(1), e44413. doi: https://doi.org/10.15517/rfl.v47i1.44413

\section{(c) $\mathbb{P Q \Theta O}$}

Doi: https://doi.org/10.15517/rfl.v47i1.44413

URL: https://revistas.ucr.ac.cr/index.php/filyling/index 
Lingüística

Fricativas y africadas en lenguas campa entre los siglos XVIII-

XX. Clasificación y diacronía de la familia campa ${ }^{1}$

Fricatives and Affricates in Kampa Languages During 18th to
20th Century. Classification and Diachrony of The Kampan
Family

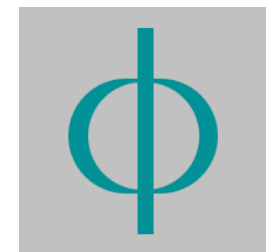

Faucet Pareja, Carlos Alberto

(iD) Carlos Alberto Faucet Pareja

carlos.faucet@unmsm.edu.pe

Universidad Nacional Mayor de San Marcos, Lima, Perú

Revista de Filología y Lingüística de la Universidad de Costa Rica

Universidad de Costa Rica, Costa Rica

ISSN: 0377-628X

ISSN-e: $2215-2628$

Periodicidad: Semestral

vol. 47, núm. 1, 2021

filyling@gmail.com

Recepción: 17 Febrero 2020

Aprobación: 21 Mayo 2020

URL: http://portal.amelica.org/ameli,

jatsRepo/125/1251597030/index.htm

DOI: https://doi.org/10.15517/rfl.v47i1.44413
Resumen: A partir de un estudio fonético y fonológico relativo a segmentos fricativos y africados en 9 materiales lingüísticos de variedades de la familia lingüística campa, recolectados in situ por expedicionarios y misioneros entre los siglos XVIII-XX, se discuten aspectos de la clasificación interna y de la diacrónica de la familia.

Palabras clave: lenguas campa, familia campa, fricativas, africadas, clasificación interna.

Abstract: On the basis of a phonetic and phonological study about fricative and affricate segmentes in 9 linguistic materials of varieties of the Kampa language family collected in field by expeditionaries and missionaries during 18th to 20th century, aspects of internal classification and diachrony of the aforementioned family are discussed.

Keywords: Kampa languages, Kampan family, fricatives, affricates, internal classification.

\section{INTRODUCCIÓN}

La familia lingüística campa es una rama nítidamente definida en el interior de la gran familia arahuaca. Su territorio tradicional se halla hacia el lado oriental de los Andes centrales, en zonas de Amazonía alta y baja que pertenecen a los departamentos peruanos de Ucayali, Huánuco, Pasco, Junín, Ayacucho, Apurímac, Cusco y Madre de Dios, si bien hay hablantes también en Loreto, Lima e incluso en Brasil (MINEDU, 2013; Michael, 2011).

En el pasado, toda variedad campa era aludida indistintamente mediante una fórmula como "el idioma de los indios antis o campas". Hoy, con una mezcla de criterios -lingüístico, político, de uso-, se hace la distinción de hasta siete "lenguas": asháninka, ashéninka, ashéninka del Gran Pajonal, kakinte, nomatsigenga, matsigenka y nanti (ver Figura 1) ${ }^{2}$. El repertorio fonológico compartido por los miembros de la familia incluye las consonantes /p, t, $\mathrm{k}, \mathrm{B}, \mathrm{s}, \int, \mathrm{h}, \mathrm{ts}, \mathrm{t}, \mathrm{m}, \mathrm{n}, \mathrm{r} /$, la semiconsonante / $\mathrm{j} / \mathrm{y}$ las vocales /a, e, i, o/. A estas se añaden la vocal / $9 /$ en nomatsigenga y los diptongos / ti / en matsigenka y / Uii/ en nanti. 


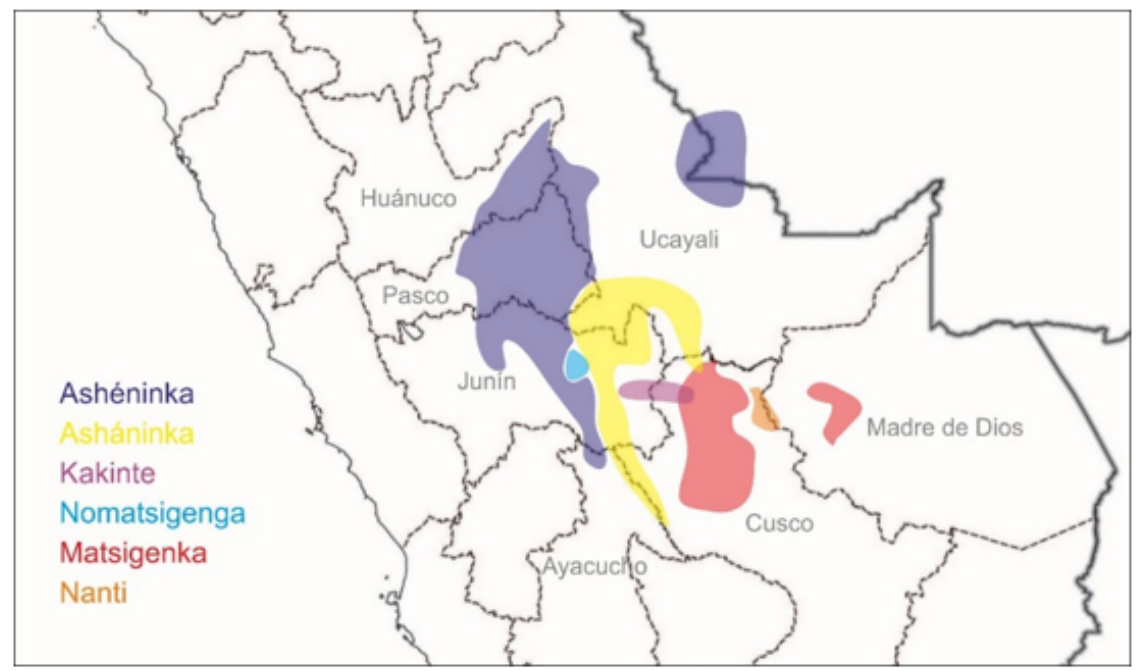

FIGURA 1.

Distribución geográfica aproximada de las lenguas campa Fuente: Elaborada a partir de Michael (2011).

Diversos materiales léxicos y gramaticales campa, recolectados in situ por expedicionarios y misioneros entre los siglos XVIII-XX, se han archivado sin ser aprovechados en los estudios sobre estas lenguas. El presente trabajo ofrece un estudio fonético y fonológico relativo a fricativas y africadas en 9 de esos materiales, con énfasis en el orden alveolar y alveoaplatal, y utiliza estos resultados para poner en discusión tanto la clasificación interna como otros aspectos de la diacronía de esta familia lingüística. La estructura del artículo es como sigue: en la primera sección, se presenta los materiales y el método utilizado para su estudio; en la segunda, se exponen los resultados obtenidos; en la tercera, se abre la discusión de estos en relación con la calidad de los materiales y con estudios diacrónicos atingentes, y en la última sección, se ofrecen las conclusiones alcanzadas.

\section{Materiales y método}

\subsection{Materiales}

En esta subsección se realiza una presentación breve de los materiales, cada uno de los cuales es denominado de acuerdo con el primer apellido del presunto transcriptor.

\subsubsection{De La Marca}

Se trata de un material catequístico hallado en Toledo (España) por Charles Leclerc y publicado en París (Francia) por Lucien Adam, en 1890, con el título Arte de la lengua de los indios Antis o Campas. Varias preguntas, advertencias y doctrina cristiana. Dirigido a lectores castellanohablantes, el material original está conformado por un "arte" gramatical, así como por textos y vocabularios que habrían sido producidos en el segundo tercio del siglo XVIII en la conversión de Sonomoro -correspondiente, al parecer, a la actual localidad de San Antonio de Sonomoro de la provincia de Satipo, departamento de Junín, en el centro del Perú-, tutelada por el sacerdote francés Juan de La Marca (Varese, 2006). Para la publicación, todo el léxico presente en los vocabularios y disperso en el resto de la obra fue recogido y reorganizado por Lucien Adam, 
quien agrupó bajo una misma entrada "básica" todas las palabras entre las cuales, según su análisis, hubiera "relación”. De acuerdo con el texto introductorio de Adam, escrito en francés, los vocabularios originales estaban desarrollados en el sentido castellano-lengua nativa.

\subsubsection{Castelnau}

Se trata de un corto material léxico recogido en 1846 y publicado en 1851 en el tomo quinto del libro Expédition dans les parties centrales de l'Amérique du Sud: de Rio de Janeiro à Lima, et de Lima au Para, a propósito de una misión científica franco-peruana dirigida, del lado francés, por Francis de Castelnau (Riviale, 2000). Designado como "Langue des antis du revèrs oriental des Andes (Echaraté)" (Castelnau, 1851, p. 290), el material habría sido recopilado en territorio de la actual provincia de Echarate, en el departamento de Cusco, en el sur del Perú. Dirigida la obra a un lector francés, las entradas del material léxico en cuestión son desarrolladas en el sentido francés-lengua nativa. El tomo contiene varios léxicos más, correspondientes a una variedad de quechua sureño y numerosas lenguas amazónicas.

\subsubsection{Marcoy}

Se trata de un corto material léxico recogido en 1846 y publicado en 1869 en el libro Voyage à travers l'Amérique du Sud, de l'Océan Pacifique à l'Océan Atlantique -pero aparecido primero en Le Tour du Monde entre 1863 y 1864 (Wise, 2017)- bajo el nombre de "Paul Marcoy", seudónimo del naturalista francés Laurent de Saint-Cricq, quien coincidiera y se acoplara a un tramo de la expedición de Francis de Castelnau (Carrasco, 1879). Dirigida la obra a un lector francés, las entradas son desarrolladas en el sentido francéslengua nativa. El libro presenta algunos léxicos más, correspondientes a otras lenguas amazónicas.

\subsubsection{Carrasco}

Parte del informe elevado al Gobierno por el militar peruano Francisco Carrasco acerca de su viaje de exploración por los ríos Vilcanota -también conocido como Santa Ana, Huillcamayo y Urubamba- y Ucayali durante el año 1846, pero publicado de manera independiente en el "Boletín de la Sociedad Geográfica de Lima" en 1901, se trata de un corto material léxico titulado "Principales palabras del idioma de las cuatro tribus de infieles que siguen" (Carrasco, 1901, p. 205), "tribus" que en el material son denominadas "antis", "piros", "conibos" y "sipibos" (sic). Las entradas son desarrolladas en el orden castellano-lengua nativa. Carrasco encabezó la parte peruana de la misión de la que Francis de Castelnau fue miembro.

\subsubsection{Zavala}

Recogido en 1891 por el militar peruano Miguel Zavala durante la exploración del río Pichis a propósito de un estudio para la prolongación del ferrocarril central y publicado en 1895 como apéndice del libro La vía central, de Joaquín Capelo, se trata de un material léxico titulado "Vocabulario de lengua campa" (Zavala, 1895, p. 155). Las entradas son desarrolladas en el sentido castellano-lengua nativa. 


\subsubsection{Delgado}

Recogido por el geógrafo peruano Eulogio Delgado durante su estadía "entre los indios Campas del río Perené" (Delgado, 1896-1897, p. 445) a propósito del estudio para la prolongación del ferrocarril central, y publicado en varios números del "Boletín de la Sociedad Geográfica de Lima”, se trata de un material léxico titulado "Vocabulario del idioma de las tribus campas" (Delgado, 1896-1897, p. 445). Las entradas son desarrolladas en el sentido castellano-lengua nativa.

\subsubsection{Sala}

Publicado en 1905 en el libro Diccionario castellano, inga, amueixa y campa por el misionero español Gabriel Sala, se trata de un material léxico multilingüe cuyas entradas son desarrolladas en el sentido sugerido en el título. Salvo la indicación de que, para 1905, la obra había estado "varios años sepultada en el archivo de la Prefectura Apostólica del Ucayali” y la de que Sala habría enfocado su labor en el "Oriente de la República” (Sala, 1905, p. 4), no hay datos acerca de la fecha y región en las que los materiales fueron recopilados. Sin embargo, se sabe que el misionero en cuestión llegó a Perú en 1868, recorrió los ríos Pichis, Pachitea y Ucayali, así como la región del Gran Pajonal, entre los años 1896 y 1897, y que murió en 1898 (Espinosa de Rivero, 2016).

\subsubsection{Touchaux}

Recogido en la "región del Apurímac, desde Simariva hasta la confluencia del Mantaro" (Touchaux, 1909, p. 3) -tramo que hoy corresponde al extremo del departamento de Cusco que limita con Ayacucho y Juníny publicado en 1909 con el título Apuntes sobre la gramática y el diccionario del idioma campa o lengua de los antis tal como se usa en el río Apurimac por el misionero francés Mauricio Touchaux, se trata de un material léxico dirigido a hablantes-escribientes de castellano, cuyas entradas son desarrolladas en el sentido castellanolengua nativa.

\subsubsection{Aza}

Recopilado "de los labios de los mismos machiguengas del Urubamba y Madre de Dios" y publicado 1924 con el título Vocabulario Español-Machiguenga (Aza Martínez de Vega, 2009 [1924]) por el misionero español José Pío Aza Martínez de Vega, se trata de un material léxico cuyas entradas son desarrolladas en el sentido castellano-lengua nativa. Aza Martínez de Vega fundó la misión San José de Koribeni en 1918, donde permaneció hasta 1921 y donde, probablemente, recogió buena parte de los materiales que luego publicaría.

La Figura 2 ofrece la ubicación geográfica aproximada ${ }^{3}$ de los lugares en los que todos los materiales presentados habrían sido recogidos. 


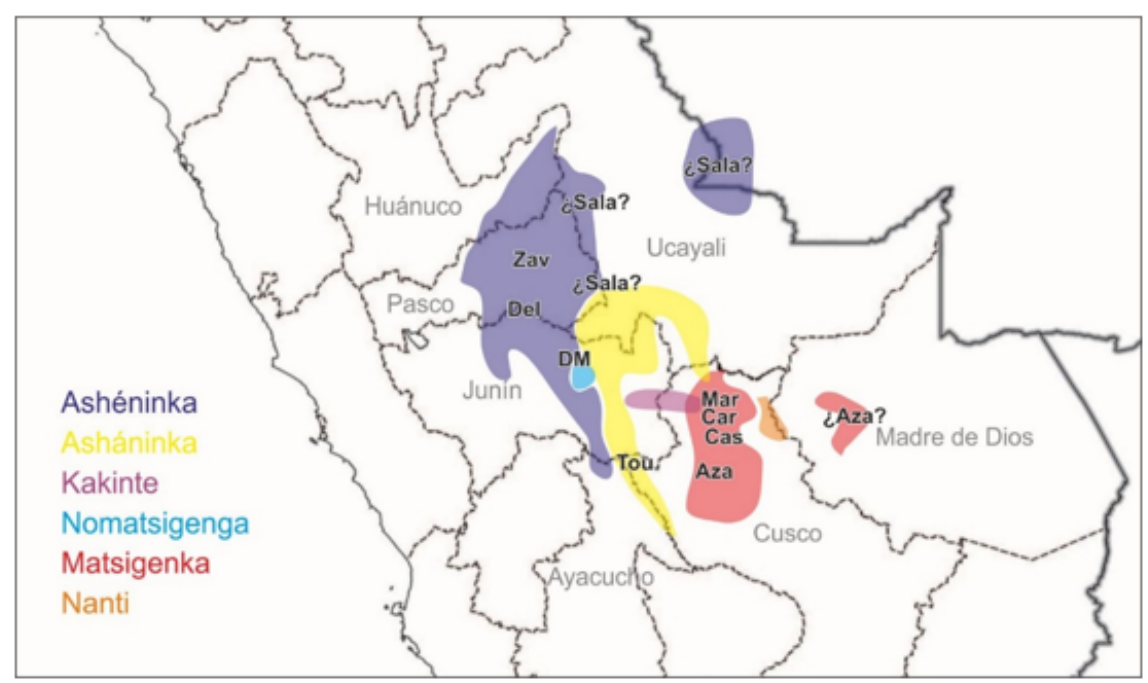

FIGURA 2 .

Ubicación geográfica aproximada de los lugares de recolección de los materiales en relación con la ubicación aproximada de las lenguas campa

\subsection{Método}

Ante datos de lenguas de sociedades ágrafas, transcritos en alguna versión del alfabeto latino antes o al margen de la lingüística moderna y sometidos, por ello, a distorsiones por las limitaciones de los transcriptores -como ocurre con los materiales enlistados arriba-, el método de análisis pertinente es el que Adolfo Constenla sistematizó y llamó “restitución” (Constenla Umaña, 2000). Adaptado aquí para su aplicación a materiales de lenguas de la misma familia, y no a dialectos o muestras de una misma lengua, el método procede de la manera que sigue. Primero, se reúnen todas las transcripciones de un mismo lema y se construye, por cada material, un lexicón organizado en relación con el castellano ${ }^{4}$. Segundo, se tabulan las grafías registradas en cada uno de los lexicones teniendo en cuenta su contexto de aparición. Tercero, se determina el valor fonético de las grafías con base en distintos criterios (reconocimiento del sistema de escritura empleado, época de recolección de los materiales, observaciones hechas por los transcriptores, conocimientos diacrónicos, areales y de universales lingüísticos, entre otros, a los que se añade aquí dos criterios más: vacilación en la escritura y comparación con transcripciones menos problemáticas del mismo autor). Cuarto, haciendo las equivalencias entre la distribución de las grafías y la de los fonos, se determinan las relaciones entre estos (distribución equivalente, complementaria, variación libre, etc.). Quinto, se establece la fonología de los materiales considerados.

\section{ANÁLISIS Y RESULTADOS}

En esta sección se ofrece el análisis de los materiales y los resultados fonéticos y fonológicos obtenidos a partir de él. Con el fin de evitar una repetición excesiva, los análisis son expuestos por grupos, en la medida en que la semejanza entre unos y otros materiales lo permite. 


\subsection{Grafías y fonética}

\subsubsection{De la Marca}

La información sobre grafías en De la Marca es detallada en las Tablas 1 y $2^{5}$.

TABLA 1.

Distribución y número de las grafías que en De la Marca corresponden a fonemas fricativos en las lenguas campa modernas

\begin{tabular}{|c|c|c|c|c|}
\hline & $-<a>$ & $\_<0, u>$ & $-\langle e, i\rangle$ & $-<i, \mathrm{e}\rangle$ \\
\hline$\langle\mathrm{s}\rangle$ & $4(2 \sim<\mathrm{tz}>)$ & 1 & $\begin{array}{l}2(1<\mathrm{s}[\mathrm{a}]>\sim \\
<\varsigma>)\end{array}$ & $2(1 \sim<\zeta$, çh $>, 1 \sim<s>)$ \\
\hline$\beta_{c}>$ & & & & 1 \\
\hline$\left\langle\zeta \xi_{\zeta}\right\rangle$ & & & $1(1 \sim<\mathrm{s}[\mathrm{a}]>)$ & $6(2 \sim<c ̧ h\rangle, 1 \sim<c h>, 1 \sim<c ̧ h, s\rangle)$ \\
\hline <çh> & 2 & 2 & $4(1 \sim<t z>)$ & $\begin{array}{l}\left.\left.16\left(2 \sim<_{\zeta}\right\rangle, 2 \sim<_{\mathrm{ch}}\right\rangle, 1 \sim<_{\zeta}, \mathrm{s}\right\rangle, 1 \\
\sim<\mathrm{s}\rangle)\end{array}$ \\
\hline$\langle\mathrm{g}\rangle$ & & & 1 & 1 \\
\hline $\mathrm{h}>$ & & & 1 & \\
\hline$\langle\mathrm{ch}\rangle$ & & & 1 & $\left.\left.3\left(1 \sim<_{\zeta}\right\rangle, 2 \sim<_{c ̧} h\right\rangle\right)$ \\
\hline$\langle\mathrm{tz}\rangle$ & $1(1 \sim<s>)$ & & $1(\sim<c ̧ h>)$ & \\
\hline
\end{tabular}


TABLA 2.

Distribución y número de las grafías que en De la Marca corresponden a fonemas africados en las lenguas campa modernas

\begin{tabular}{|c|c|c|c|c|}
\hline & $\_<a>$ & ${ }_{-}^{<0, u>}$ & $\_\langle e, i\rangle$ & $\_i, e>$ \\
\hline$\langle t\rangle$ & $3(1 \sim<\operatorname{ch}[\mathrm{e}]>)$ & 8 & 3 & 13 \\
\hline$<\mathrm{z}>$ & $2(1 \sim<\mathrm{tz}\rangle)$ & & & \\
\hline$\langle\mathrm{tz}\rangle$ & $\begin{array}{l}5(1 \sim<\mathrm{CZ}>, 2 \sim \\
\langle\mathrm{z}>)\end{array}$ & $4(1 \sim<\mathrm{ch}>)$ & & \\
\hline$\varepsilon_{\mathrm{CZ}}>$ & 1 & & & \\
\hline$u_{\zeta}>$ & & & & $1(1 \sim<\mathrm{ch}>)$ \\
\hline <çh> & & 1 & & 3 \\
\hline$\langle\mathrm{ch}>$ & 3 & $2(1 \sim<\mathrm{tz}>)$ & $1(1 \sim<\mathrm{t}[\mathrm{a}]>)$ & $\begin{array}{l}11(1 \sim<\mathrm{ch}, \mathrm{cch}>, 1 \sim \\
\left.<_{\zeta}>\right)\end{array}$ \\
\hline$<\mathrm{cch}>$ & & & & $1(1 \sim<\mathrm{tch}, \mathrm{ch}>)$ \\
\hline$\langle\operatorname{tch}\rangle$ & & & & $2(1 \sim<\operatorname{ch}, \mathrm{cch}>)$ \\
\hline
\end{tabular}

Los valores fonéticos $\mathrm{de}<\mathrm{s}>\mathrm{y}<\mathrm{c} h>$ son descritos en el "arte"6 como correspondientes a los fonos fricativos alveolar sordo [s] y alveopalatal sordo [ $]$, descripción que concuerda con la situación actual en las lenguas campa, que poseen un fonema $/ \mathrm{s} / \mathrm{y}$ otro $/ \mathrm{S} /$. Para las demás grafías, ante la falta de información fonética de parte del autor, la comparación con las lenguas modernas y la fluctuación en la escritura de un mismo lema indican lo siguiente: $\langle\mathrm{t}\rangle$ representaría un fono alveolar sordo $[\mathrm{t}] ;\langle\mathrm{c}\rangle$ y $\langle$ ç $\rangle-$ si es que la única muestra de $\langle\mathrm{c}\rangle$ no consiste en una errata por $\langle c ̧\rangle-$, uno alveolar [s], como en francés; $\langle\mathrm{g}\rangle \mathrm{y}\langle\mathrm{h}\rangle$, uno fricativo glotal sordo $[\mathrm{h}] ;\langle\mathrm{tz}\rangle,\langle\mathrm{z}\rangle \mathrm{y}\langle\mathrm{cz}\rangle-$ si el único caso de esta última no es una errata por $\langle\mathrm{tz}\rangle-$, uno africado alveolar sordo $[\mathrm{ts}]^{7} ;<\mathrm{ch}>$, uno africado palatal sordo [t] , como en castellano; $\mathrm{y}<\mathrm{cch}>\mathrm{y}<\mathrm{tch}>$, uno africado alveolar sordo palatalizado, semejante pero no idéntico a $[\mathrm{t}]$, acaso $\left[\mathrm{ts}^{\mathrm{j}}\right]$. La última conclusión surge de las siguientes observaciones: primero, el trígrafo está conformado por una letra asociada con la oclusión, más un dígrafo asociado con la africación y estridencia, recursos que en conjunto redundan en la representación de una africada para un lector castellanohablante, público objetivo del material original; segundo, la restricción del trígrafo al contexto de las vocales /e/ y /i/ apunta a un carácter palatal en el fono representado; y tercero, el intercambio con $<\mathrm{ch}>$ en diferentes muestras de un mismo lema indica que dichos trígrafos representan un fono muy semejante a $[\mathrm{t}]$.

Trasladando esta información a las Tablas 1 y 2, se tendría que los dos fonos fricativos orales, [s] y [ $]$, estarían en distribución equivalente ante la vocal /a/ -obsérvese que no son intercambiados en ningún lemay, en menor medida, ante /o/ y /e/; pero estarían en variación libre ante /i/, contexto en el cual el segundo fono es el más frecuente en los lemas que no muestran fluctuación. Los dos fonos africados, [ts] y [ $\mathrm{t}]$ ], estarían 
en distribución defectiva: ambos aparecen en el contexto de /a/ -no son intercambiados en ningún lema-y en menor medida en el de /o/; pero no en el de /e/ ni en el de /i/, vocales ante las cuales solo aparece [ $\mathrm{t}$ ], acompañado excepcionalmente por el otro fono africado sordo, palatal o palatalizado, acaso [ts'], que parece estar en distribución complementaria con [ts]. Como conjunto, los fonos fricativos orales mostrarían una ligera tendencia a fluctuar con los africados: en la posición de inicio de palabra, $[\mathrm{s}]$ variaría con [ts] ante /a/ $\mathrm{y}[\mathrm{J}]$ con $[\mathrm{t}]$ ante /e/; mientras que en interior, $\left[\int\right]$ variaría con $[\mathrm{t}]$ ante /e/ y /i/. La variación de africados hacia fricativos sería excepcional, en cambio. En cuanto a [h], este parece estar en distribución defectiva con los fricativos orales. Por último, hay un indicio muy poco convincente de la existencia de fluctuación entre $[\mathrm{t}]$ y un fono oclusivo dentoalveolar sordo $[\mathrm{t}] \mathrm{o}\left[\mathrm{t}^{\mathrm{h}}\right]$.

\subsubsection{Castelnau, Marcoy y Carrasco}

Las Tablas 3 y 4 ofrecen la información sobre las grafías en los tres autores ${ }^{8}$.

TABLA 3.

Distribución y número de las grafías en Castelnau, Marcoy y Carrasco que corresponden a fonemas fricativos en las lenguas campa modernas

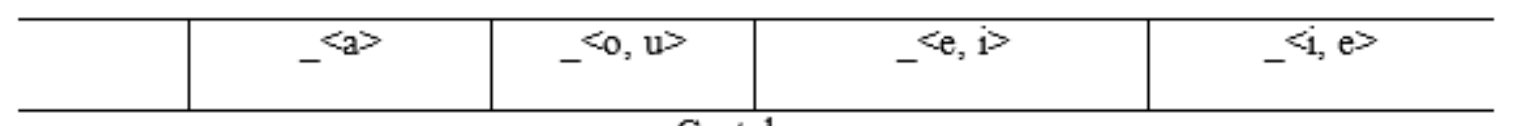

\section{Castelnau}

\begin{tabular}{l|l|l|l|l}
\hline$<_{\mathrm{S}}>$ & 1 & 2 & $\begin{array}{l}12(1<\mathrm{s}[\mathrm{io}]>, 1 \\
\left.<_{\mathrm{s}[\mathrm{ia}]}>\right)\end{array}$ \\
\hline
\end{tabular}

Marcoy

\begin{tabular}{l|l|l|l|l}
\hline$<\mathrm{s}>$ & 2 & & $2(1<\mathrm{s}[\mathrm{ia}]>, 1<\mathrm{s}[\mathrm{ai}]>)$ & $10(1<\mathrm{s}[\mathrm{ia}]>)$ \\
\hline$<\mathrm{gh}>$ & & & 1 & \\
\hline$<\mathrm{ch}>$ & & & & 1 \\
\hline
\end{tabular}

Carrasco

\begin{tabular}{l|l|l|l|l}
\hline$<\mathrm{s}>$ & 3 & 1 & $3(1<\mathrm{s}[\mathrm{ie}]>)$ & 13 \\
\hline$<\mathrm{sch}>$ & & & 1 & \\
\hline$<\mathrm{s}>$ & 1 & & & \\
\hline$<\mathrm{g}>$ & & & 1 & \\
\hline$<\mathrm{ch}>$ & 1 & & & 1 \\
\hline
\end{tabular}


TABLA 4.

Distribución y uso de las grafías en Castelnau, Marcoy y Carrasco que corresponden a fonemas africados en las lenguas campa modernas

\begin{tabular}{l|c|c|c|c}
\hline & $-<\mathrm{a}>$ & $-<\mathrm{o}, \mathrm{u}>$ & $-<\mathrm{e}, \mathrm{i}>$ & $-<\mathrm{i}, \mathrm{e}>$ \\
\hline
\end{tabular}

Castelnau

\begin{tabular}{l|l|l|l|l}
\hline$<\mathrm{t}>$ & 3 & 7 & & 7 \\
\hline$<\mathrm{st}>$ & & & & 2 \\
\hline$<\mathrm{ts}>$ & 1 & 2 & & 1 \\
\hline$<\mathrm{t}-\mathrm{s}>$ & & & 1 & \\
\hline$<\mathrm{ch}>$ & 3 & 1 & & 7 \\
\hline$<\mathrm{tch}>$ & & 1 & & \\
\hline
\end{tabular}

Marcoy

\begin{tabular}{l|l|l|l|l}
\hline$<\mathrm{t}>$ & 1 & 6 & & 11 \\
\hline$<\mathrm{ts}>$ & 1 & & & \\
\hline$<\mathrm{ch}>$ & 5 & 3 & & 9 \\
\hline
\end{tabular}

Carrasco

\begin{tabular}{l|l|l|l|l}
\hline$<>$ & 2 & 4 & & 13 \\
\hline$<\mathrm{ch}>$ & 8 & $4(1<\mathrm{ch}[\mathrm{i}]>)$ & & 11 \\
\hline
\end{tabular}

En ninguno de los materiales hay información fonética. Dado que la comparación con las lenguas modernas sugiere la posibilidad de que una sola grafía esté infrarrepresentando a dos fonos -en los tres materiales, $<s>$ podría representar al fono fricativo alveolar sordo $[\mathrm{s}]$ o tanto a este como al fricativo alveopalatal sordo [ $\int$; y exclusivamente en Carrasco, la grafía $<\mathrm{ch}>$, al fono africado palatal sordo $[\mathrm{t}]$ o tanto a este como al africado alveolar sordo [ts]-, la determinación de los valores fonéticos será guiada, aquí, por la revisión de otros léxicos recogidos en las mismas fuentes. Así, las tablas ${ }^{9} 5$ y 6 entregan correlaciones entre las grafías para fricativas y africadas en los léxicos "pano", "conibo" y "sipibo" ${ }^{10}$ también presentes en Castelnau (1851), Marcoy (1869) y Carrasco (1901) y los fonemas a los que dichas grafías corresponden en las lenguas shipibo (Loriot, Lauriault y Day, 1993) y kapanawa (Loos y Loos, 2003) de la familia pano. 
Revista de Filología y Lingüística de la Universidad de Costa Rica, 2021, 47(1), Enero-Junio, ISSN...

TABLA 5

Correlación de las grafías y contextos en Castelnau, Marcoy

y Carrasco con los fonemas fricativos en shipibo y kapanawa

\begin{tabular}{|c|c|c|c|c|}
\hline \multirow{2}{*}{ Contexto } & \multirow{2}{*}{$\begin{array}{l}\text { Castelnau } \\
\text { "langue des panos" }\end{array}$} & \multirow{2}{*}{$\begin{array}{c}\text { Marcoy } \\
\text { "idiome conibo" }\end{array}$} & \multicolumn{2}{|c|}{ Carrasco } \\
\hline & & & de los "conibos" & de los "sipibos" \\
\hline \multicolumn{5}{|c|}{ Shipibo /s/, kapanawa /s/' } \\
\hline$-\langle a\rangle$ & $\langle 9\rangle$ & & & \\
\hline$-\langle 0, u\rangle$ & $\langle 99>(1)$ & $\langle 99\rangle$ & $\langle 99\rangle$ & $\langle 99\rangle$ \\
\hline$-\langle e\rangle$ & & & $\langle 9\rangle$ & $\langle 9\rangle$ \\
\hline$-\langle i, e\rangle$ & & & $\langle 9\rangle$ & $\langle 9\rangle$ \\
\hline$-\#, \$$ & $<[\mathrm{i}] \mathrm{sh}>(1)$ & $\langle 9\rangle$ & $\langle 9\rangle$ & $\langle 9\rangle$ \\
\hline \multicolumn{5}{|c|}{ Shipibo / / / , kapanawa $/ / /$} \\
\hline$-^{\langle a\rangle}$ & $\begin{array}{l}\langle g r\rangle,\langle g h\rangle,\langle s\rangle, \\
<g h r\rangle\end{array}$ & $\langle 9\rangle$ & $\langle 9\rangle,\langle 55\rangle,\langle r\rangle$ & $\langle 9\rangle,\langle 55\rangle,\langle\gamma\rangle$ \\
\hline$-\langle 0, \mathrm{~d}\rangle$ & & $\begin{array}{l}\langle r\rangle,\langle 5\rangle,\langle s h\rangle, \\
\langle r s\rangle\end{array}$ & $\begin{array}{l}\langle r\rangle,\langle c 5\rangle,\langle 5\rangle,\langle 55\rangle, \\
\langle r s\rangle\end{array}$ & $\langle r|\langle c 5\rangle(1),\langle 5\rangle(1)$ \\
\hline$-\langle\theta\rangle$ & $\begin{array}{l}\langle r s\rangle,\langle s c h\rangle,\langle s h\rangle, \\
\langle s c\rangle\end{array}$ & $\langle 9\rangle,\langle 5 h\rangle$ & $\langle 9\rangle$ & $\langle 99\rangle$ \\
\hline$-\langle i, e>$ & & & $\langle r\rangle$ & er \\
\hline$-\#, \$$ & $\operatorname{sgh}\rangle$ & $\langle 9\rangle,\langle c h\rangle,\langle 99\rangle$ & $\langle r\rangle,\langle 9\rangle,\langle 55\rangle$ & $\langle r\rangle,\langle 9\rangle,\langle 55\rangle$ \\
\hline \multicolumn{5}{|c|}{ Shipibo / / , kapanawa //f? } \\
\hline$-\langle\beta\rangle$ & & & $\langle 9 c h\rangle(1)$ & $\langle g c h\rangle(1)$ \\
\hline$-\langle 0, u\rangle$ & $\langle\mathrm{ch}\rangle(1)$ & & & \\
\hline \multicolumn{5}{|l|}{$-<e>$} \\
\hline$-\langle i, e\rangle$ & $\langle\mathrm{ch}\rangle(3)$ & $\langle r\rangle,\langle x\rangle(1)$ & $\begin{array}{l}\langle\mathrm{ch}\rangle,\langle 9\rangle(1),\langle\text { sich }\rangle \\
\text { (1) }\end{array}$ & $\begin{array}{l}\langle\mathrm{ch}\rangle,\langle 9\rangle(1),\langle\text { sich }\rangle \\
\text { (1) }\end{array}$ \\
\hline$-\#, \$$ & $\langle 9\rangle(1)$ & $\langle q\rangle(1),<[\mathrm{i}] \gg(1)$ & $\langle\mathrm{ch}\rangle(2),\langle[\mathrm{i}] \mathrm{s}>(2)$ & $\langle\mathrm{ch}\rangle(2),\langle[\mathrm{i}] \mathrm{s}\rangle(2)$ \\
\hline
\end{tabular}


TABLA 6.

Correlación de las grafías y contextos en Castelnau, Marcoy

y Carrasco con los fonemas africados en shipibo y kapanawa

\begin{tabular}{l|c|c|c|l}
\hline \multirow{2}{*}{ Contexto } & Castelnau & Marcoy & \multicolumn{2}{|c}{ Carrasco } \\
\cline { 3 - 4 } & "langue des & "idiome conibo" & de los "conibos" & de lo "sipibos" \\
& panos" & & & \\
\hline
\end{tabular}

Shipibo/t/, kapanawa / $/ \mathrm{s}^{\prime}$

\begin{tabular}{|c|c|c|c|c|}
\hline - $\langle a\rangle$ & $\langle\mathrm{ts}\rangle(1)$ & $\langle\mathrm{ds}\rangle(1)$ & $\langle\mathrm{ts}\rangle(1)$ & $\langle[\#] \mathrm{s}>(1)$, ts $(2)$ \\
\hline$-\langle 0, \mathrm{u}\rangle$ & & & $\langle\operatorname{cs}\rangle(1)$ & $\langle\operatorname{cs}\rangle(1)$ \\
\hline$-\langle e\rangle$ & & & & \\
\hline$-\langle i, e\rangle$ & $\langle\mathrm{ch}\rangle(1)$ & $\langle\mathrm{ch}\rangle(1)$ & $\begin{array}{l}\langle s c h\rangle(1),\langle c h\rangle \\
\text { (2) }\end{array}$ & $\begin{array}{l}\langle s c h\rangle(1),\langle\operatorname{ch}\rangle \\
\text { (2) }\end{array}$ \\
\hline
\end{tabular}

Shipibo $/ \mathrm{g} /$, kapanawa $/ \mathrm{g} /$

\begin{tabular}{l|l|l|l|l}
\hline$-<\mathrm{a}>$ & $<\mathrm{ch}>(1)$ & $<\mathrm{ch}>(2)$ & $<\mathrm{ch}>(2)$ \\
\hline$-<0, \mathrm{u}>$ & & $<\mathrm{ch}>(2)$ & & $<\mathrm{ch}>(1)$ \\
\hline$-<\mathrm{e}>$ & & & & \\
\hline$-<\mathrm{i}, \mathrm{e}>$ & $<\mathrm{ch}>(3)$ & $<\mathrm{ch}>(5)$ & $<\mathrm{ch}>(9)$ & $<\mathrm{ch}>(8)$ \\
\hline
\end{tabular}

Del lado de las fricativas, a pesar de la diversidad en la representación de la retrofleja /ş/, en la Tabla 5 se observa que casi no hay solapamiento entre las grafías que corresponden a /s/y las que corresponden a $/ \mathrm{J} /$, lo que autoriza a afirmar que, respecto del léxico pano, en los tres materiales habría una distinción relativamente clara entre una fricativa alveolar y una alveopalatal y que estas serían representadas por diferentes grafías básicas: $\langle s\rangle$ para la primera $\mathrm{y}\langle\mathrm{ch}\rangle,\langle\mathrm{sch}\rangle-$ a la manera francesa- y otras para la segunda ${ }^{11}$. Del lado de las africadas, la correlación de la Tabla 6 es más nítida: el fonema africado palatal sordo / $\mathrm{t} /$ corresponde en los tres materiales a $<\mathrm{ch}>-$ considérese que la lengua materna de Castelnau y Marcoy fue el francés, que no posee africadas, de modo que estos habrían percibido el fono [ $\mathrm{t}]$ como $\left[\int\right] \mathrm{y}$, consecuentemente, lo habrían representado mediante $<\mathrm{ch}>-$, mientras que el africado alveolar sordo $/ \mathrm{ts} /$, a $<$ ts $>$ y grafías diferentes de $<\mathrm{ch}>$, excepto ante la vocal /i/, contexto en el cual figuran en Castelnau y Marcoy y $<\mathrm{ch}>\mathrm{y}<\mathrm{sch}>$ en Carrasco -en lo que parece responder a una palatalización de /ts/ ante /i/ claramente percibida y anotada por los tres autores-.

Retornando con estas conclusiones al material campa de las Tablas 3 y 4, y considerando la comparación con las lenguas campa modernas, la grafía $\langle\mathrm{t}\rangle$ representaría un fono oclusivo alveolar sordo $[\mathrm{t}] ;\langle\mathrm{j}\rangle,\langle\mathrm{g}\rangle$ $\mathrm{y}\langle$ gh $>$, uno fricativo glotal sordo $[\mathrm{h}] ;\langle\mathrm{s}\rangle \mathrm{y}\langle\mathrm{sch}\rangle$, los fonos fricativos alveolar sordo [s] y alveopalatal sordo [ $]$, respectivamente, en los tres materiales; $\langle\mathrm{ch}\rangle$, uno alveopalatal sordo [S] en los dos primeros y otro africado palatal sordo [ $\mathrm{t}$ ] en los tres; $\mathrm{y}\langle\mathrm{ts}\rangle,\langle\mathrm{st}\rangle \mathrm{y}\langle\mathrm{t}-\mathrm{s}\rangle$, a uno africado alveolar sordo [ts], registrado en Castelnau y Marcoy, pero no en Carrasco. En Castelnau, la grafía $<\mathrm{tch}>$ también representaría un fono [ts]. 
En los tres materiales, el fono [s] es el que figura en el mayor número de contextos, incluido el de la vocal / i/; [ $]$ solo aparece ante esta última y, exclusivamente en Carrasco, también ante /e/, pero siempre de manera excepcional $^{12}$. En Castelnau, [ts] y [ $\mathrm{t}$ ] estarían en distribución equivalente en el contexto de /a/, /o/ e /i/, con una tendencia a variar libremente ante la última vocal. En Marcoy, estarían en distribución defectiva: ambos aparecen ante /a/, pero [ $\mathrm{t}]$ ] es el único ante /i/. En Carrasco, en cambio, [ $\mathrm{t}$ ] es el único africado que figura en todo el léxico, ante todas las vocales. Asi mismo, el fono [h] aparece solo en Marcoy y Carrasco. Por último, no hay indicios de variación entre $[\mathrm{t}]$ y $[\mathrm{t}]$.

\subsubsection{Zavala, Delgado y Sala}

La información sobre grafías en Zavala (1895) es ofrecida en las Tablas 7 y 8.

TABLA 7.

Distribución y uso de las grafías en Zavala que corresponden a fonemas fricativos en las lenguas campa modernas

\begin{tabular}{|c|c|c|c|c|}
\hline & $-<a>$ & $-<0, u>$ & $-\langle e, p$ & $-<i, e>$ \\
\hline$\langle j\rangle$ & 2 & & 1 & \\
\hline$<\mathrm{z}>$ & & 1 & & \\
\hline$\langle s\rangle$ & 1 & & & 1 \\
\hline$\langle$ ss $\rangle$ & & & & $1(1 \sim<\mathrm{ssh}>)$ \\
\hline$<\mathrm{sh}>$ & & & $5(1 \sim<\mathrm{ch}>)$ & $15(1<\operatorname{sh}[$ ui $]>\sim<\operatorname{ssh}[$ ui $]>)$ \\
\hline$<$ ssh $>$ & & & & $1(1<\mathrm{ssh}[\mathrm{ui}]>\sim<\operatorname{sh}[\mathrm{ui}]>, 1 \sim<\mathrm{ss}\rangle)$ \\
\hline$<\mathrm{ch}>$ & & & $1(1 \sim<\operatorname{sh}>)$ & \\
\hline
\end{tabular}


TABLA 8.

Distribución y uso de las grafías en Zavala que corresponden a fonemas africados en las lenguas campa modernas

\begin{tabular}{l|l|l|l|l}
\hline & \multicolumn{1}{|c|}{$-<\mathrm{a}>$} & $-<\mathrm{o}, \mathrm{u}>$ & $-<\mathrm{e}, \mathrm{p}>$ & \multicolumn{1}{c}{$-<\mathrm{i}, \mathrm{e}>$} \\
\hline$<\mathrm{t}>$ & 7 & 10 & & 1 \\
\hline$<\mathrm{sh}>$ & & & & $3(2 \sim<\mathrm{ch}>)$ \\
\hline$<\mathrm{st}>$ & & & & 1 \\
\hline$<\mathrm{ts}>$ & & & & $12(1 \sim<\mathrm{tsh}>, 2 \sim<\mathrm{ch}>)$ \\
\hline$<\mathrm{tsh}>$ & & & & $1(1 \sim<\mathrm{ts}>)$ \\
\hline$<\mathrm{tch}>$ & 1 & 2 & & $12(2 \sim<\mathrm{sh}>, 2 \sim<\mathrm{ts}>)$ \\
\hline$<\mathrm{ch}>$ & & & & 1 \\
\hline$<\mathrm{csh}>$ & & & & \\
\hline
\end{tabular}

Zavala no presenta información fonética sobre las grafías utilizadas. Sin embargo, la comparación con las lenguas modernas y la vacilación en la escritura de un mismo lema indican que $\langle\mathrm{t}\rangle$ representaría un fono oclusivo dentoalveolar sordo $[\mathrm{t}] ;\langle\mathrm{s}\rangle$, uno fricativo alveolar sordo $[\mathrm{s}] ;\langle\mathrm{sh}\rangle \mathrm{y}\langle\mathrm{z}\rangle$, uno fricativo alveopalatal sordo $[\mathrm{S}] ;<\mathrm{ss}>\mathrm{y}\langle\mathrm{ssh}>$, uno o varios fonos fricativos sordos, alveopalatales o palatalizados, acaso $[\mathrm{s}] ;<\mathrm{j}>$, uno fricativo glotal sordo $[\mathrm{h}]$; $\langle$ st $>$ y $\langle$ ts $>$, uno africado alveolar sordo $[\mathrm{ts}] ;<\mathrm{ch}\rangle$, uno africado palatal sordo $[\mathrm{t}] \mathrm{y}$, marginalmente, quizá también uno africado alveolar sordo [ts]; $\mathrm{y}\langle\mathrm{tsh}\rangle,\langle\mathrm{tch}\rangle \mathrm{y}\langle\mathrm{csh}\rangle$, un fono africado dentolaveolar sordo palatalizado [ts'], o diferentes fonos próximos a este, intermedios entre [ts] y $[\mathrm{t}]$ ]. La conclusión del valor fonético de $<\mathrm{ss}>\mathrm{y}<\mathrm{ssh}\rangle$ surge de las siguientes observaciones: primero, estas grafías están constituidas por dos letras $<s>$ juntas al inicio, duplicación que para un hablante-escribiente de castellano relativamente moderno, como lo fue Zavala, puede representar insistencia en un sonido con salida de aire sibilante ligeramente estridente, pero no idéntico ni a $[s]$ ni a $\left[\int\right]$-de ahí la necesidad de acuñar nuevas grafías-; segundo, $<$ ss $>$ y $<$ ssh $>$ están limitadas al contexto de /i/, lo que apunta a un carácter palatal en los sonidos representados; y tercero, el intercambio entre ellas y con $\langle$ sh $\rangle$ en diferentes muestras de un mismo lema señala que su valor fonético es semejante al de $<\mathrm{sh}\rangle$. La conclusión del valor de $<$ tsh $>$, $<\mathrm{tch}>\mathrm{y}<\mathrm{csh}>$ procede de las observaciones siguientes: primero, estos trígrafos $-\mathrm{si}<\mathrm{csh}>$ no es una errata por $<$ tsh $>-$ están constituidos por una letra asociada con la oclusión, más un dígrafo asociado con la salida de aire estridente, recursos que en conjunto configuran la representación de una africada para un hablanteescribiente de castellano, como lo fue Zavala; segundo, la restricción de $<\mathrm{tsh}>\mathrm{y}<\mathrm{csh}>$ al contexto de la vocal /i/ apunta a un carácter palatal en el fono representado; y tercero, en Zavala, los lemas <catcharini $>$ y $<$ catcha> 'humo', que son los que portan la grafía <tch>, se corresponden con / katSiari/ en nomatsigenga (Shaver, 1996) y /katfa:ri/ en ashéninka (Payne, 1980), par de lexemas cuya comparación revela un cambio en las africadas con una hipotética pero muy probable etapa intermedia [ts'], que justificaría la acuñación y vacilación alrededor de un trígrafo que representara tal fono africado inexistente en la lengua materna de Zavala, semejante a $[\mathrm{ts}]$ y a $[\mathrm{t}]$, pero no idéntico a ninguno de estos. 
Revista de Filología y Lingüística de la Universidad de Costa Rica, 2021, 47(1), Enero-Junio, ISSN...

En cuanto a Delgado (1896-1897), la información pertinente es entregada en las Tablas 9 y 10.

TABLA 9.

Distribución y uso de las grafías en Delgado que corresponden a fonemas fricativos en las lenguas campa modernas

\begin{tabular}{|c|c|c|c|c|}
\hline & $\_<a$ & $\_<0, u>$ & $\left.\_<\mathrm{e}, \mathrm{p}\right\rangle$ & $-\langle i, e\rangle$ \\
\hline \multicolumn{5}{|c|}{ Delgado } \\
\hline$\langle s\rangle$ & 1 & & 1 & 1 \\
\hline$\langle$ ss $\rangle$ & & 1 & & \\
\hline$<\operatorname{sh}>$ & 1 & 1 & 5 & 16 \\
\hline$<\mathrm{g}\rangle$ & & & 1 & \\
\hline$\langle\mathrm{h}>$ & & & & 1 \\
\hline$<\mathrm{tsh}>$ & & & $1\left(\# \_\right)$ & \\
\hline$\langle\mathrm{tz}\rangle$ & & & & $3\left(1 \# \_\right)$ \\
\hline
\end{tabular}


TABLA 10.

Distribución y uso de las grafías en Delgado que corresponden a fonemas africados en las lenguas campa modernas

\begin{tabular}{|c|c|c|c|c|}
\hline & $-\langle a\rangle$ & $-\langle 0, u>$ & $\left.{ }_{-}<\mathrm{e}, \mathrm{i}\right\rangle$ & $-\langle i, e\rangle$ \\
\hline \multicolumn{5}{|c|}{ Delgado } \\
\hline$\langle t>$ & $4(1 \sim<t z>)$ & 5 & 1 & $8(5 \sim<\mathrm{tz}\rangle, 1 \sim<\mathrm{ch}\rangle)$ \\
\hline$\langle\mathrm{tt}\rangle$ & & & & 1 \\
\hline$<$ th $>$ & & 1 & & \\
\hline$<$ sh $>$ & $2(1 \#)$ & $1(1 \#)$ & & $4(2 \#,, 1 \sim<\mathrm{ch}, \mathrm{st}>)$ \\
\hline$\langle$ st $>$ & & & & $1(1 \sim<\mathrm{ch}, \mathrm{st}\rangle)$ \\
\hline$\langle\mathrm{ts}\rangle$ & $2(1 \sim<\mathrm{tz}\rangle)$ & & & 1 \\
\hline$\langle\operatorname{tsh}\rangle$ & & & & 1 \\
\hline$\langle\mathrm{tz}\rangle$ & $\begin{array}{l}4(1 \sim\langle\mathrm{ts}\rangle, 1 \sim \\
\langle\mathrm{t}\rangle)\end{array}$ & 2 & $1(1 \sim<\mathrm{tch}>)$ & $\begin{array}{l}13(5 \sim<\mathrm{t}>, 1 \sim<\mathrm{ch}>, 1 \sim \\
<\mathrm{tch}>)\end{array}$ \\
\hline$\langle$ tztz $>$ & & & & $1(1 \sim \operatorname{tzch}>)$ \\
\hline$<\mathrm{tzch}>$ & & & & $1(1 \sim<\mathrm{tztz}>)$ \\
\hline$<\mathrm{ch}>$ & 1 & & & $\begin{array}{l}7(1 \sim<\mathrm{tz}\rangle, 1 \sim<\mathrm{sh}, \mathrm{st}\rangle, 1 \sim \\
\langle\mathrm{t} \gg)\end{array}$ \\
\hline$\langle\mathrm{tch}\rangle$ & & & $1(1 \sim<\mathrm{tz}>)$ & 1 \\
\hline
\end{tabular}

En relación con Delgado, el valor fonético de $<\mathrm{sh}>$ es descrito como correspondiente a un fono fricativo alveopalatal sordo $\left[\int\right]^{13}$. Para las demás grafías, la comparación con las lenguas modernas y la vacilación en la escritura de un mismo lema indican que $\langle\mathrm{t}\rangle \mathrm{y}\langle\mathrm{tt}\rangle-$ si la única muestra del último no es una erratarepresentarían un fono oclusivo dentoalveolar sordo $[\mathrm{t}]$, pero $\langle\mathrm{t}\rangle$ también uno oclusivo dentoalveolar sordo aspirado $\left.\left[\mathrm{t}^{\mathrm{h}}\right] ;<\mathrm{th}\right\rangle$, también el oclusivo dentoalveolar sordo aspirado $\left[\mathrm{t}^{\mathrm{h}}\right] ;\langle\mathrm{s}\rangle$, uno fricativo alveolar sordo $[\mathrm{s}]$; $\langle\mathrm{ss}\rangle$, uno oclusivo sordo, ligeramente palatalizado, quizá intermedio entre $[\mathrm{s}] \mathrm{y}\left[\int\right]$; $\langle\mathrm{g}\rangle \mathrm{y}\langle\mathrm{h}\rangle$, uno glotal sordo [h]; $\langle$ st $\rangle$, $\langle\mathrm{ts}\rangle \mathrm{y}\langle\mathrm{tz}\rangle$, uno africado alveolar sordo [ts]; $\langle\mathrm{ch}\rangle$, uno africado palatal sordo [t] ; $\mathrm{y}\langle\mathrm{tsh}\rangle,\langle\mathrm{tztz}\rangle,\langle\mathrm{tzch}\rangle \mathrm{y}\langle\mathrm{tch}\rangle$, uno africado alveolar sordo palatalizado [ts'], o varios fonos semejantes a este, intermedios entre [ts] y [t $]$. La conclusión acerca del valor de $<s s>$ se basa en la observación de que el dígrafo está constituido por dos letras $<s>$ juntas, duplicación que para un hablante-escribiente de castellano relativamente moderno, como lo fue Delgado, puede representar insistencia en un sonido con salida de aire sibilante ligeramente estridente, pero no idéntico ni a $[\mathrm{s}]$ ni a $\left[\int\right]$-de ahí la necesidad de acuñar nuevas 
grafías-. La conclusión respecto del valor de $\langle$ tsh $\rangle,\langle\mathrm{tztz}\rangle,\langle\mathrm{tzch}\rangle \mathrm{y}\langle\mathrm{tch}\rangle$ está basada en las siguientes observaciones: primero, la serie de grafías está constituida por una letra asociada con la oclusión más un dígrafo asociado con la estridencia, o por dos dígrafos que representan africadas, secuencias que configuran o redundan en la representación de un fono africado para un hablante-escribiente de castellano, como lo fue Delgado; y segundo, la serie se halla restringida a aparecer en el contexto de las vocales anteriores /e/ y /i/, lo que apunta a un carácter palatal en el fono o fonos representados.

En relación con Sala, la información atingente es ofrecida en las Tablas 11 y 12.

TABLA 11.

Distribución y uso de las grafías en Sala que corresponden a fonemas fricativos en las lenguas campa modernas

\begin{tabular}{|c|c|c|c|c|}
\hline & $<<a>$ & $-<0, u>$ & $\_<e, p$ & $\_i, e>$ \\
\hline$\langle s\rangle$ & $1(1 \sim \#<t z>)$ & 1 & 1 & $1(1 \sim<\operatorname{ch}>)$ \\
\hline$<\mathrm{sh}>$ & & & & $3(2 \sim<\mathrm{ch}>)$ \\
\hline$<$ sch $>$ & & & & $7(4 \sim<\operatorname{ch}>)$ \\
\hline$<\mathrm{g}\rangle$ & & & & 1 \\
\hline$\langle j\rangle$ & 1 & & & \\
\hline$\langle\mathrm{ts}\rangle$ & & & $1(1 \sim<\mathrm{ch}>)$ & \\
\hline$\langle\mathrm{tz}\rangle$ & $1(1 \sim<s>)$ & & & $1(\#)$ \\
\hline$<\mathrm{ch}>$ & 1 & 1 & $\begin{array}{l}8(1 \sim<\mathrm{ts}\rangle, 1 \sim \\
<\mathrm{chs}>)\end{array}$ & $\begin{array}{l}15(4 \sim<\mathrm{sch}>, 2 \sim<\mathrm{sh}>, 1 \sim \\
<\mathrm{s}>)\end{array}$ \\
\hline <chs $>$ & & & $1(1 \sim<\mathrm{ch}>)$ & \\
\hline
\end{tabular}


TABLA 12.

Distribución y uso de las grafías en Sala que corresponden a fonemas africados en las lenguas campa modernas

\begin{tabular}{|c|c|c|c|c|}
\hline & - $\langle a\rangle$ & _ $\langle 0, \mathrm{u}\rangle$ & ${ }_{-}\langle\mathrm{e}, \mathrm{p}\rangle$ & $\_\langle i, e\rangle$ \\
\hline$\langle t\rangle$ & $\begin{array}{l}7(4 \sim<\mathrm{tz}\rangle, 1 \sim<\mathrm{cz}, \\
\mathrm{ts}\rangle, 1 \sim\langle\mathrm{tt}\rangle, 1 \sim \\
\langle\mathrm{ts}\rangle)\end{array}$ & $\begin{array}{l}15(1 \sim<\mathrm{tz}\rangle, 1 \\
\sim<\mathrm{t}, \mathrm{ts}\rangle, 1 \sim \\
<\mathrm{tt}>)\end{array}$ & $\begin{array}{l}1 \quad(1 \sim \\
<\mathrm{ch}>)\end{array}$ & $\begin{array}{l}4(1 \sim\langle\mathrm{ts}\rangle, 2 \sim<\mathrm{ch}\rangle, 1 \sim<\mathrm{st}, \\
\mathrm{ch}\rangle)\end{array}$ \\
\hline$\langle\mathrm{tt}\rangle$ & $1(1 \sim<t>)$ & $1(1 \sim<\mathrm{t}>)$ & & \\
\hline$\langle\operatorname{sh}\rangle$ & & & & $2(1 \sim\langle\mathrm{ts}, \mathrm{ch}\rangle)$ \\
\hline$\langle$ st $\rangle$ & & & & $1(1 \sim<\mathrm{t}, \mathrm{ch}>)$ \\
\hline$\langle z\rangle$ & & $1(1 \sim\langle\mathrm{tz}\rangle)$ & & \\
\hline$\langle\mathrm{tz}\rangle$ & $8(4 \sim<t>)$ & $\begin{array}{l}4(1 \sim<\mathrm{t}\rangle, 1 \sim \\
<\mathrm{t}, \mathrm{t}\rangle, 1 \sim<\mathrm{z}\rangle)\end{array}$ & & $1(1 \sim<\mathrm{ch}>)$ \\
\hline$\langle\mathrm{cz}\rangle$ & $1(1 \sim\langle t, t s\rangle)$ & & & \\
\hline$\langle\mathrm{ts}\rangle$ & $2(1 \sim<\mathrm{ch}\rangle)$ & $1(1 \sim\langle\mathrm{tz}, \mathrm{t}\rangle)$ & & $\begin{array}{l}5(1 \sim<\mathrm{tt}>, 1 \sim<\mathrm{ch}\rangle, 1 \sim<\mathrm{sh}, \\
\mathrm{ch}>, 2 \sim<\mathrm{tch}>, 1 \sim<\mathrm{ch}, \mathrm{tch}>)\end{array}$ \\
\hline$\langle\mathrm{ch}\rangle$ & $3(1 \sim\langle\mathrm{ts}\rangle)$ & 1 & $1(1 \sim<t>)$ & $\begin{array}{l}19(1 \sim\langle\mathrm{ts}\rangle, 1 \sim\langle\mathrm{tz}\rangle, 2 \sim<\mathrm{t}\rangle, \\
1<\mathrm{ch}[\text { ia }, \mathrm{a}]\rangle, 1 \sim<\mathrm{ts}, \mathrm{tch}\rangle, 1 \sim \\
\langle\mathrm{tch}\rangle, 1 \sim<\mathrm{t}, \mathrm{st}\rangle)\end{array}$ \\
\hline$\langle\mathrm{tch}\rangle$ & & & & $\begin{array}{l}4(2 \sim\langle\mathrm{ts}\rangle, 1 \sim<\mathrm{ts}, \mathrm{ch}\rangle, 1 \sim \\
<\mathrm{ch}\rangle)\end{array}$ \\
\hline
\end{tabular}

En Sala, no hay información fonética explícita acerca de las grafías. La comparación con las lenguas modernas y la vacilación en la escritura de los lemas indica que $\langle\mathrm{t}\rangle$ representaría un fono oclusivo dentoalveolar sordo $[\mathrm{t}]$, pero también uno oclusivo dentoalveolar sordo aspirado $\left[\mathrm{t}^{\mathrm{h}}\right] ;\langle\mathrm{tt}\rangle$, uno oclusivo dentoalveolar sordo $[\mathrm{t}] ;\langle\mathrm{s}\rangle$, uno fricativo alveolar sordo $[\mathrm{s}] ;\langle\mathrm{sh}\rangle,\langle\mathrm{sch}\rangle \mathrm{y}\langle\mathrm{chs}\rangle$, uno fricativo alveopalatal sordo $\left[\int\right] ;\langle$ g $\rangle$ y $\langle$ j $\rangle$, uno fricativo glotal sordo $[\mathrm{h}] ;\langle\mathrm{z}\rangle,\langle\mathrm{tz}\rangle,\langle\mathrm{ts}\rangle,\langle$ st $\rangle$ y $\langle\mathrm{cz}\rangle-$ si las dos últimas no son erratas-, uno africado alveolar sordo $[\mathrm{ts}] ;<\mathrm{ch}>$, uno africado alveolar sordo $[\mathrm{t}]$, pero también uno fricativo alveopalatal $\left[\int\right]$-a la manera francesa, en este último caso-; y $\langle$ tch $>$, uno africado alveolar sordo palatalizado $\left[\mathrm{ts}^{\mathrm{j}}\right]$, intermedio entre $[\mathrm{ts}]$ y $[\mathrm{t}]$. Esta conclusión surge también de la observación de que $\langle\mathrm{tch}\rangle$ se halla restringido al contexto palatal de $/ \mathrm{i} / \mathrm{y}$ es intercambiado con los fonos representados por $\langle\mathrm{ts}\rangle \mathrm{y}<\mathrm{ch}\rangle$ en muestras del mismo lema ${ }^{14}$.

Trasladando todas las equivalencias a las Tablas 7-12, se tiene que, en los tres materiales, [s] -parcialmente reemplazado por $[\mathrm{h}]$ en inicio de palabra en Zavala y Sala- y $\left[\int\right]$ parecen estar en distribución defectiva: 
ambos aparecen ante /a/, pero [ $\left.\int\right]$ es el único de los dos que aparece ante /e/ y virtualmente el único de los dos ante /i/, contexto en el cual las excepcionales muestras de [s] fluctúan con [ $\left.\int\right]$ en Zavala y en Sala. Asimismo, $[\mathrm{ts}]$ y $[\mathrm{t}]$ estarían en distribución equitativa ante todas las vocales en los tres materiales, pero con matices: primero, $[\mathrm{ts}]$ es reemplazado parcialmente o fluctúa con $\left[\mathrm{t}^{\mathrm{h}}\right]$ en el contraste con $[\mathrm{t}]$ ante la vocal /a/ y/o ante la vocal /o/, según cada material; segundo, hay cierta variación libre entre ellos y [tsi] en el contexto de las vocales /e/ y/o/ /i/, según cada material. También en los tres materiales, parece haber una excepcional fluctuación entre fonos fricativos y africados en la posición de inicio de palabra, fluctuación que no puede ser recuperada completamente en Sala, dado que aquí <ch> puede representar tanto a la fricativa [S] como a la africada $[\mathrm{t}]$, como fue señalado líneas arriba. Por último, exclusivamente en Delgado y Sala, [t] está en variación libre con las africadas $[\mathrm{ts}] \mathrm{y}[\mathrm{t}]$ ante /i/.

\subsubsection{Touchaux y Aza}

En relación con las grafías en Touchaux y Aza, las Tablas 13 y 14 entregan la información pertinente.

TABLA 13.

Distribución y uso de las grafías en Touchaux y Aza que corresponden a fonemas fricativos en las lenguas campa modernas

\begin{tabular}{|c|c|c|c|c|}
\hline & $-\langle a\rangle$ & $-\langle 0, u\rangle$ & $-\langle e\rangle$, & $-\langle i, e\rangle$ \\
\hline \multicolumn{5}{|c|}{ Touchaux } \\
\hline$\langle s\rangle$ & 4 & 1 & 5 & $8(4 \sim<\operatorname{sh}>)$ \\
\hline$\langle\operatorname{sh}\rangle$ & 1 & & & $13(4 \sim\langle s\rangle, 1<\operatorname{sh}[i \circ]\rangle, 1<\operatorname{sh}[\mathrm{ie}]\rangle)$ \\
\hline$\langle\mathrm{ch}\rangle$ & & & & 2 \\
\hline \multicolumn{5}{|c|}{ Aza } \\
\hline$\langle s\rangle$ & 5 & $1(1 \sim\langle\mathrm{ts}\rangle)$ & 7 & $8(5 \sim\langle\mathrm{sh}\rangle, 1 \sim\langle\mathrm{ts}\rangle)$ \\
\hline$\langle\operatorname{sh}\rangle$ & 1 & & & $\begin{array}{l}24(5 \sim\langle s\rangle, 2 \sim\langle\mathrm{ch}\rangle, 1<\operatorname{sh}[\mathrm{ie}]\rangle, 1 \\
\langle\operatorname{sh}[\mathrm{io}]\rangle)\end{array}$ \\
\hline$\langle\mathrm{h}\rangle$ & $1(1 \sim<j>)$ & & $1(1 \sim\langle j\rangle)$ & \\
\hline$\langle j\rangle$ & $1(1 \sim\langle h\rangle)$ & & $1(1 \sim\langle\mathrm{h}\rangle)$ & \\
\hline \multicolumn{5}{|l|}{$\langle\mathrm{ts}\rangle$} \\
\hline$\langle\operatorname{ch}\rangle$ & & & & $2(2 \sim<\operatorname{sh}>)$ \\
\hline
\end{tabular}


TABLA 14.

Distribución y uso de las grafías en Touchaux y Aza que corresponden a fonemas africados en las lenguas campa modernas

\begin{tabular}{l|r|r|r|r}
\hline & $-<a\rangle$ & $-<0, \mathrm{u}\rangle$ & $-\langle\mathrm{e}, \mathrm{p}\rangle$ & $\left.{ }_{-}<\mathrm{i}, \mathrm{e}\right\rangle$ \\
\hline
\end{tabular}

\begin{tabular}{|c|c|c|c|c|}
\hline$\langle\mathrm{t}\rangle$ & 3 & $9(1 \sim\langle\mathrm{ts}\rangle, 1 \sim<\mathrm{ts}, \mathrm{ch}\rangle)$ & 3 & 14 \\
\hline$\langle s\rangle$ & 1 & & & \\
\hline$\langle z\rangle$ & & $1(1 \sim<\mathrm{ts}\rangle)$ & & \\
\hline$\langle\mathrm{ts}\rangle$ & $3(1 \sim<\mathrm{ch}>)$ & $\begin{array}{l}4(1 \sim<\mathrm{t}\rangle, 1 \sim<\mathrm{z}\rangle, 1 \sim<\mathrm{t}, \mathrm{ch}\rangle, \\
1 \sim<\mathrm{ch}\rangle)\end{array}$ & 1 & 1 \\
\hline$\langle\mathrm{ch}\rangle$ & $6(1 \sim\langle\mathrm{ts}\rangle)$ & $4(1 \sim<\mathrm{t}, \mathrm{ts}\rangle, 1 \sim\langle\mathrm{ts}\rangle)$ & 1 & $14(1<\operatorname{ch}[$ ia $]\rangle)$ \\
\hline
\end{tabular}

Aza

\begin{tabular}{|c|c|c|c|c|}
\hline$\langle\mathrm{t}\rangle$ & 2 & 8 & 2 & 14 \\
\hline$\langle\beta\rangle$ & $6(2 \sim\langle\mathrm{ts}\rangle)$ & $5(1 \sim\langle s h, t s, t z\rangle)$ & & \\
\hline$\langle\operatorname{sh}\rangle$ & & $1(1 \sim<\mathrm{s}, \mathrm{ts}, \mathrm{tz}\rangle)$ & & $1(1 \sim<\mathrm{sh}\rangle)$ \\
\hline$\langle t z\rangle$ & & $1(1 \sim\langle s, s h, t s\rangle)$ & & \\
\hline$\langle\mathrm{ts}\rangle$ & $3(1 \sim<\mathrm{ch}, \mathrm{CH}\rangle, 2 \sim<\mathrm{s}\rangle)$ & $1(1 \sim\langle s, s h, t z\rangle)$ & & \\
\hline$\langle\mathrm{ch}\rangle$ & $8(1 \sim<\mathrm{ts}, \mathrm{CH}\rangle, 1<\mathrm{ch}[1]\rangle)$ & & & $15(1 \sim<\mathrm{sh}>)$ \\
\hline$\langle\mathrm{CH}\rangle$ & $1(1 \sim<\mathrm{ts}, \mathrm{ch}>)$ & & 1 & \\
\hline
\end{tabular}

En ambos materiales, el valor fonético de $<$ sh $>$ es descrito explícitamente como correspondiente a un fono fricativo alveopalatal sordo $\left[\int\right]^{15}$. Para las demás grafías, la comparación con las lenguas modernas y la vacilación en la escritura de los lemas indican que la grafía $<\mathrm{t}>$ representaría un fono oclusivo dentoalveolar sordo $[\mathrm{t}]$ y, solo en Touchaux, también uno oclusivo dentoalveolar sordo aspirado $\left[\mathrm{t}^{\mathrm{h}}\right] ;\langle\mathrm{s}\rangle$, uno fricativo alveolar sordo $[\mathrm{s}] ;\langle\mathrm{h}\rangle \mathrm{y}\langle\mathrm{j}\rangle$, uno fricativo glotal sordo $[\mathrm{h}] ;\langle\mathrm{ch}\rangle$, uno africado palatal sordo $[\mathrm{t}]^{16} ;\langle\mathrm{z}\rangle$, $<\mathrm{ts}>\mathrm{y}<\mathrm{tz}>$, uno africado alveolar sordo [ts]; y exclusivamente en Aza, $\langle\mathrm{s}\rangle \mathrm{y}<\mathrm{CH}\rangle$, también uno africado alveolar sordo $[\mathrm{ts}]^{17}$.

Según las equivalencias establecidas, $[s]$ y $\left[\int\right]$ estarían en distribución equitativa en ambos materiales en el contexto de /a/ - no son intercambiados en ningún lema- y en el de /i/, aunque con tendencia a variar libremente entre sí ante esta última vocal; y [ts] y [ $\mathrm{t}]$ estarían en distribución equitativa ante /a/ y /o/, pero complementaria ante /e/ y /i/, pues el primero es el único de los dos ante /e/, mientras que el segundo, el único ante $/ \mathrm{i} /{ }^{18}$. Asimismo, las fricativas orales variarían libremente con las africadas en la posición de inicio de palabra: $[\mathrm{s}]$ con $[\mathrm{ts}]$ y $\left[\int\right]$ con $[\mathrm{t}]$, tendencia que no es observada en las africadas. En cuanto a $[\mathrm{h}]$, solo Aza ofrece muestras de este fono ante /a/ y ante /e/. Por último, en ninguno de los materiales hay variación 
entre $\left[\mathrm{t}\right.$ ] y algún fono africado ante /i/, aunque en Touchuax sí habría una variación libre entre [ $\mathrm{t}^{\mathrm{h}}$ ], [ts] y $[\mathrm{t}]$ ante $/ \mathrm{o} /$, único contexto en el que aparece $\left[\mathrm{t}^{\mathrm{h}}\right]$.

\subsection{Fonología}

Apartando el reemplazo parcial de $[\mathrm{s}]$ por $[\mathrm{h}]$ y de $[\mathrm{ts}]$ por $\left[\mathrm{t}^{\mathrm{h}}\right]$ en algunos materiales, la distribución de los fonos $[\mathrm{s}],\left[\int\right],[\mathrm{ts}]$ y $[\mathrm{t}]$ sugiere la presencia generalizada de los fonemas $/ \mathrm{s} /, / \mathrm{s} / \mathrm{/} / \mathrm{ts} / \mathrm{y} / \mathrm{t} /$, excepto en Castelnau, Marcoy y Carrasco, pues en estos solo habría indicios claros de la existencia de /s/; y especialmente en el último, donde el único fonema africado aparente sería $/ \mathrm{t} /$. Asimismo, en todos los materiales habría un fonema $/ \mathrm{h} / \mathrm{y}$ un fonema $/ \mathrm{t} /$, excepto en Castelnau, donde no hay indicios del primero.

En particular sobre los fonemas $/ \mathrm{s} /, / \mathrm{J} / \mathrm{t} / \mathrm{ts} / \mathrm{y} / \mathrm{t} /$, se observa que su rendimiento es desigual a través del espectro de materiales. Así, la aparición exclusiva o mayoritaria de $\left[\int\right]$ ante las realizaciones de /e/ y de / i/ en De la Marca, Zavala y Delgado sugiere que en estos los únicos fonemas ante dichas vocales serían / $/$; mientras que en el resto de materiales que presentan las oposiciones en cuestión, la fluctuación entre $[s]$ y $\left[\int\right]$ y entre $[\mathrm{ts}]$ y $[\mathrm{t}]$ sugiere que los fonemas serían $/ \mathrm{s} / \mathrm{y} / \mathrm{ts} /$, realizables en los fonos $[\mathrm{S}] \mathrm{y}[\mathrm{t}]$ como producto de una palatalización motivada por la coarticulación con las realizaciones de tales vocales. Sin embargo, mientras que esta conclusión no es problemática respecto de las fricativas, sí lo es respecto de las africadas (ver 4.2).

\subsection{Síntesis de los resultados}

La Figura 3 sintetiza la correlación entre grafías, fonos y fonemas establecida aquí para cada uno de los materiales. 


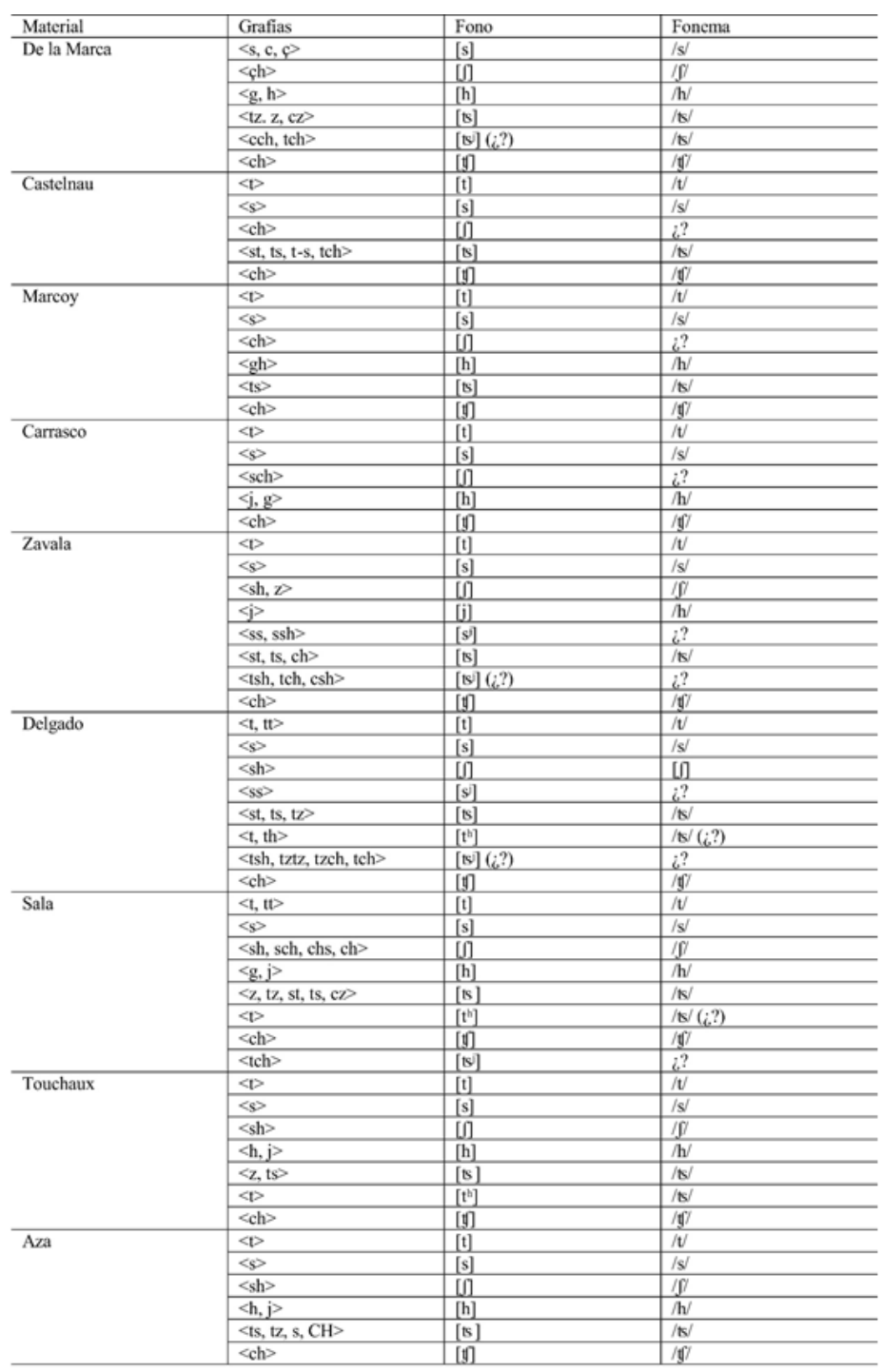

FIGURA 3.

Correlación de grafías, fonos y fonemas en cada uno de los materiales

\section{Discusión}

\subsection{Confiabilidad de los materiales}

El bajo número de entradas en Castelnau, Marcoy y Carrasco sugiere relativizar los resultados obtenidos a partir de ellos, especialmente el de la ausencia de un fono $\left[\int\right]$ y un fonema $/ \int /$ así como, exclusivamente en Carrasco, el de la ausencia de un fono [ts] y un fonema /ts/. Más aun, el hecho de que los tres autores realizaran juntos parte del viaje en el que recogieron los léxicos campa y pano abre la posibilidad de que estos no correspondieran a transcriptores y colaboradores independientes, sino a uno solo, lo que redunda en contra de la confiabilidad de los materiales en la medida en que alguno de los autores podrían haberse limitado a reproducir las anotaciones de otro. No obstante, hay dos argumentos en favor de los resultados: primero, cada uno de los tres materiales exhibe características particulares, que sugieren que los colaboradores, o por lo menos los transcriptores, habrían actuado de manera independiente ${ }^{19}$; y segundo, siendo hablantes nativos de francés, Francis de Castelnau y Paul Marcoy (Laurent de Saint-Cricq) podían percibir y anotar con recursos 
gráficos inequívocos un fono [ $\left.\int\right]$-como quedó claro a partir de la revisión de los otros léxicos transcritos por ellos-, de manera que, si ellos hubieran escuchado un fono de esa naturaleza en el léxico campa, no cabe duda de que este podría ser recuperado sistemáticamente de los materiales -pero no es el caso- ${ }^{20}$. El último argumento, sin embargo, no mitiga las reservas alrededor de las africadas alveolares, respecto de las cuales ninguno de los autores poseía la capacidad nativa de percibir.

\subsection{Repercusiones diacrónicas}

\subsubsection{Alrededor de la clasificación interna de la familia campa}

Todas las variedades campa actuales poseen los fonemas $/ \mathrm{s} / \mathrm{y} / \mathrm{J} /$, pero con diferencias en la distribución: en el nomatsigenga, /s/ es el único de los dos en el contexto de /i/; mientras que en las demás variedades, $/ \mathrm{J} /$ es el que aparece ante dicha vocal (Heitzman, 1975). Este panorama condujo a Michael (2011) a considerar que habría habido una innovación compartida* $/ \mathrm{s} />/ \mathrm{S} /$ i i en la historia de las variedades campa y a ensayar a partir de ella una clasificación interna de la familia. Así, de acuerdo con este autor, el cambio fonológico * / s/ > / /_i habría originado la escisión inicial del protocampa en dos variedades: una, experimentadora del cambio, cuya diversificación progresiva, guiada por otros cambios (ver Figura 4), terminaría convirtiéndola en el nanti, matsigenka, asháninka, ashéninka y kakinte; y la otra, inafecta respecto del primer cambio, que eventualmente se convertiría en el nomatsigenga actual.

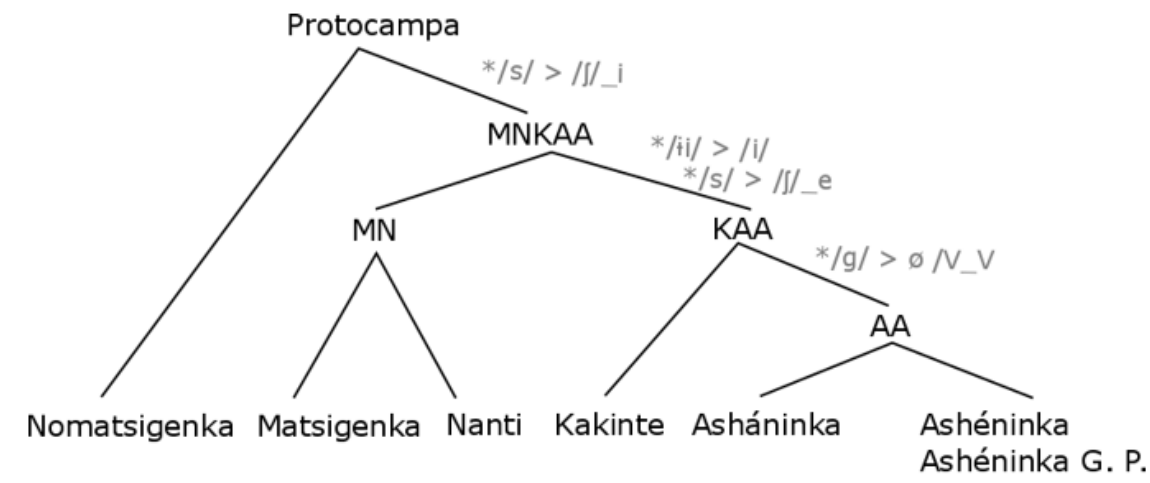

FIGURA 4.

Clasificación interna de la familia campa según Michael (2011)

Sin embargo, los resultados obtenidos aquí no apoyan la propuesta de Michael. Que [ $\left.\int\right]$ predominara numéricamente sobre [s] ante las realizaciones de /i/ hacia 1750 en De la Marca (aproximadamente, ashéninka o ashéninka del Gran Pajonal; ver abajo), pero compartiera el contexto con [s] de manera más equilibrada hacia 1890 en Touchaux (aproximadamente, kakinte o asháninka) y, no obstante, estuviera ausente en Castelnau (aproximadamente, matsigenka o nanti), Marcoy y Carrasco (aproximadamente, kakinte o asháninka) hacia 1850 , sugiere que la presencia excluyente de $/ \mathrm{S} /$ ante $/ \mathrm{i} /$ en la mayoría de variedades campa actuales no sería producto de un antiguo cambio terminado $* / \mathrm{s} />/ \mathrm{S} / \mathrm{i}$ en una protolengua MNKAA, ni siquiera de la etapa inicial de una innovación tal en esta protolengua, sino de varios cambios de ese tipo, ocurridos cada cual en su tiempo, incluso muy recientemente, en las variedades ya individualizadas. A pesar de ello, por su presencia categórica en De la Marca, Zavala, Delgado y Sala, el cambio $* / s />/ \int /$ i aún puede ser postulado como innovación compartida en las variedades representadas en estos materiales, localizadas hacia el norte del territorio campa (ver Figura 2). 
No obstante, la revisión global de los materiales sí parece respaldar la postulación del cambio * / +ii/> /i/ como la innovación compartida más antigua ${ }^{21} \mathrm{y}$, a partir de esta, una propuesta de clasificación interna de la familia campa como la esbozada en la Figura 4. Junto con la localización aproximada de estas variedades, la confluencia de los resultados de esta innovación compartida con los cambios en marcha */s/ > / $/ \_i, *$ / $\mathrm{s} />/ \int /$ e e ${ }^{*} / \mathrm{g} />\varnothing / \mathrm{V}_{-} \mathrm{V}^{22}$ permite, además, una identificación parcial de los materiales de la manera que sigue: Castelnau y Aza, como matsigenka o nanti; Marcoy, Carrasco y Touchaux, como kakinte o asháninka; y De la Marca, Zavala, Delgado y Sala, como ashéninka o ashéninka del Gran Pajonal (ver Figura 5) ${ }^{23}$.

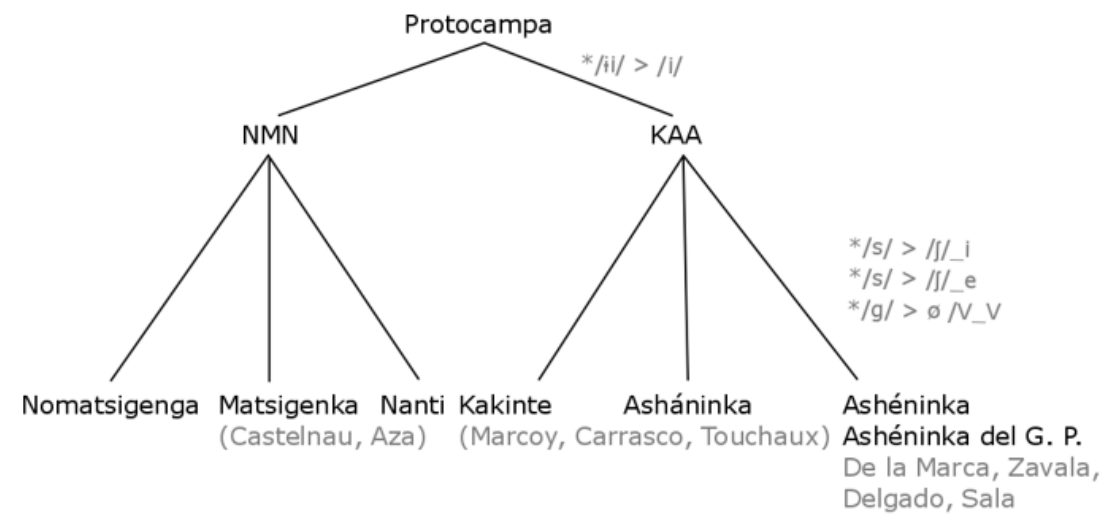

FIGURA 5.

Nuevo esbozo de clasificación interna de la familia campa

\subsubsection{Alrededor de las africadas /ts/ $y$ /tJ/ ante /i/}

En las variedades campa actuales, los fonemas /ts/ y / $\mathrm{t} /$ no contrastan en el contexto de /i/, vocal ante la cual solo aparece uno de los dos fonemas, con raras excepciones (ver Heitzman, 1975). Puesto que los resultados del presente trabajo registran fluctuación entre $[\mathrm{ts}]$ y $[\mathrm{t}]$ y/o neutralización del contraste en ese contexto en la mayoría de los materiales, la conclusión preliminar sería que el protofonema habría sido */ts/ y que este habría cambiado a / $\mathrm{t} /$ en algunas variedades como producto de una palatalización motivada por la coarticulación con /i/. No obstante, hay datos que problematizan esta explicación. Primero, Payne (1991), quien reconstruye un contraste entre $* / \mathrm{ts} / \mathrm{y} * / \mathrm{t} /$ ante $* / \mathrm{i} /$ en el protoarahuaco, arguye que, aunque aparentemente contraintuitivo en sentido articulatorio, un cambio o cambios */ts/ > $/ \mathrm{t} /$ i habrían obrado en las lenguas campa como producto de una tendencia a la fricativización de obstruyentes ante /i/ en esta familia, tendencia que estaría reflejada en el proceso morfofonológico $/ \mathrm{t} / \rightarrow[\mathrm{ts}] / \mathrm{i}$ en ashéninka del Pichis (Payne, 1991), así como en el proceso fonológico -o acaso cambio consumado- / $t / \rightarrow[\mathrm{ts}] /$ i en diferentes variedades de ashéninka y alguna de asháninka (ver Heitzman, 1975), encontrado aquí en Delgado y Zavala. Segundo, sus particularidades léxicas y su ubicación geográfica aproximada indican que la lengua registrada en Aza puede ser identificada como el matsigenka o el nanti de inicio del siglo XX. Sin embargo, en Aza solo se registra / $\mathrm{t} /$ ante /i/, mientras que en matsigenka (Snell, 2008) y nanti (Michael, 2011) actuales solo hay $/ \mathrm{ts} /$ en ese contexto. Por tanto, si el cambio antiguo hubiera sido $* / t s />/ t 5 /$ i, habría que suponer que, luego de este, cuyo resultado sería atestiguado en Aza, habría habido un cambio $/ \mathrm{t} />/ \mathrm{ts} /$ ante $/ \mathrm{i} /$ que revirtiera las cosas y diera cuenta del estado actual del matsigenka/nanti, en una secuencia de cambios más conjetural que el solo cambio $* / \mathrm{t} />/ \mathrm{ts} /$ i propuesto por Payne. Tercero, el registro de la variación [tsi] $\sim[\mathrm{t} \mathrm{i}]$ en matsigenka, respecto de una palabra desusada en el habla cotidiana, pero vigente en la tradición 
oral ([nantsi] [nant $\mathrm{i}]$ 'hermano (de mujer)' en Renard-Casevitz y Pacaia (2004, p. 46), concuerda mejor con la idea de que el cambio fuera $* / \mathrm{t} />/ \mathrm{ts} / \_\mathrm{i}$, pues, si las innovaciones fonológicas regulares se difunden a través del léxico de acuerdo con la frecuencia de uso de este -primero en el más frecuente, luego en el menos frecuente (Bybee, 2012)-, una palabra en desuso que muestra una secuencia[ $\mathrm{t} \mathrm{i}$ ] , en una lengua que en la actualidad exhibe solo / ts/ ante /i/, comportaría evidencia de que la forma anterior del fonema / $\mathrm{ts} /$ fue $/ \mathrm{t} /$, no tanto de un proceso fonológico sincrónico $/ \mathrm{ts} / \rightarrow / \mathrm{t} /$ _ i. Por todo lo anterior, los resultados obtenidos aquí invitan a no asumir fácilmente que el fonema protocampa ante $/ \mathrm{i} /$ fuera $* / \mathrm{ts} /$ en todos los casos o que este hubiera experimentado un cambio */ts/ $/ \mathrm{t} \mathrm{t} /$ _ i en algunas variedades, como podría parecer a priori; sino a considerar que el estado actual de cosas podría ser producto de cambios $* / t />/$ ts/_i relativamente recientes.

\section{CONCLUSIONES}

El desarrollo del presente trabajo permite llegar a las conclusiones que siguen, la última de las cuales es de naturaleza metodológica:

1. Los expedicionarios y misioneros que recopilaron material lingüístico campa entre los siglos XVIII-XX habrían utilizado diferentes grafías para representar los fonos oclusivo alveolar sordo $[\mathrm{t}]$, fricativo alveolar sordo $[\mathrm{s}]$, fricativo alveopalatal sordo $\left[\int\right]$ y fricativo palatal sordo $[\mathrm{t}]$, así como los fonos, no registrados por todos, oclusivo alveolar sordo aspirado $\left[\mathrm{t}^{\mathrm{h}}\right]$, fricativo glotal sorda [h], africado alveolar sordo [ts] y dos fonos de carácter palatal no claramente determinado: aproximadamente, fricativo alveolar sordo palatalizado $\left[s^{j}\right]$ y africado alveolar sordo palatalizado $\left[\mathrm{ts}^{\mathrm{j}}\right]$.

2. Con la excepción de Carrasco, todas las variedades registradas en los materiales habrían poseído los fonemas fricativos orales $/ \mathrm{s} / \mathrm{y} / \mathrm{\int} /$, así como los africados $/ \mathrm{ts} / \mathrm{y} / \mathrm{t} /$.

3. En el contexto de la vocal/i/, sin embargo, no todas estas variedades habrían presentado los fonemas $/ \mathrm{S} / \mathrm{y} / \mathrm{ts} /$.

4. La actual y bastante generalizada presencia de los fonemas $/ \int / \mathrm{y} / \mathrm{ts} /$ ante la vocal $/ \mathrm{i} / \mathrm{en}$ la familia campa sería producto no de herencia desde alguna protolengua, sino de cambios $* / s />/ \mathrm{S} / \mathrm{i}$ $y^{*} / \mathrm{t} />/ \mathrm{ts} /$ i ocurridos tardíamente en cada variedad, excepto en las variedades de ashéninka y ashéninka del Pajonal, las que, al corresponderse con De la Marca, Zavala, Delgado y Sala, sí parecen haber experimentado un cambio antiguo $* / s />/ \int /$ i.

5. Aunque recogidos de manera ingenua, los materiales lingüísticos de siglos anteriores pueden ser aprovechados para controlar las hipótesis del razonamiento diacrónico basado en materiales modernos.

\section{Bibliografía}

Adam, L. (1890). Arte de la lengua de los indios Antis o Campas. Varias preguntas, advertencias y doctrina cristiana. París, Francia: J. Maisonneuve.

ArcGis Online. (s. f.). [software]. Recuperado de https://www.esri.com/en-us/arcgis/products/arcgis-online/overview

Aza Martínez de Vega, J. (2009 [1924]). Vocabulario Español-Machiguenga. En C. García de Tuñón Aza, J. M. García de Tuñón Aza, y R. A. Ordieres (Eds.), Obras completas. Estudios Históricos, Etnográficos y Lingüisticos sobre el Sur de la Amazonía Peruana (pp. 253-382). Cusco, Perú: Centro Bartolomé de las Casas.

Beier, C. (2017). Pi-naajunyaa ikiituwaaka kuwasiini: Vamos a escribir el idioma iquito. Recuperado de http://www. cabeceras.org/beier_et_al_2017_p+-naajuuyaa-ikiituwaaka-kuwasiini_pnik.pdf 
Bybee, J. (2012). Patterns of lexical diffusion and articulatory motivation for sound change. En M. Solé, y D. Recasens (Eds.), The Initiation of Sound Change: Perception, production, and social factors (pp. 211-234). Amsterdam, Holanda: John Benjamins.

Castelnau, F. (1851). Expédition dans les parties centrales de l'Amérique du Sud: de Rio de Janeiro à Lima, et de Lima au Para. (Tomo quinto). París, Francia: P. Bertrand.

Carrasco, F. (1879). Viaje por los Ríos Huillcamayo y parte del Ucayali, ejecutado por órden del Gobierno del Perú por el Capitán de Fragata D. Francisco Carrasco. En A. Raymondi (Ed.), El Perú (vol. 3, pp. 154-192). Lima, Perú: Imprenta del Estado.

Carrasco, F. (1901). Principales palabras del idioma de las tribus de infieles que siguen. Boletín de la Sociedad Geográfica de Lima, 11, 204-211.

Constenla Umaña, A. (2000). La restitución: un método lingüístico reconstructivo sincrónico. Revista de Filología y Lingüistica, 26(2), 161-180.

Delgado, E. (1896-1897). Vocabulario del idioma de las tribus campas. Boletín de la Sociedad Geográfica de Lima, (5), 445-457; (6), 96-105, 230-240, 347-356, 393-396.

Duff-Tripp, M. (1998). Diccionario yanesha' (amuesha)-castellano. Lima: ILV.

Espinosa de Rivero, O. (2016). Los asháninkas y la violencia de las correrías durante y después de la época del caucho. Bulletin de l'Institut français d'études andines, 45(1), 137-155. Recuperado de http://journals.openedition.org/ bifea/7891

Fleck Zuazo, D., Uaquí Bëso, F. y Jiménez Huamán, D. (2012). Diccionario Matsés-Castellano. Iquitos, Perú: Tierra Nueva.

Heitzman, A. (1975). Correspondencia entre ciertos grupos de fonemas entre varios dialectos campa. En Actas del XXXIX Congreso Internacional de Americanistas, (vol. 5, pp. 165-179).

Kindberg, L. (1980). Diccionario asháninca. Yarinacocha: ILV.

Loos, E. (2008). Las lenguas pano y su reconstrucción. En O. Shell (Ed.), Estudios Panos III (pp. 11-212). Lima, Perú: ILV.

Loos, E. y Loos, B. (2003). Diccionario capanahua-castellano. Lima: ILV.

Loriot, J., Lauriault, E. y Day, D. (1993). Diccionario shipibo-castellano. Yarinacocha: ILV.

Marcoy, P. (1869). Voyage à travers l'Amérique du Sud, de l'océan Pacifique a l'océan Atlantique (primer tomo). París, Francia: Hachette.

Michael, L. (2011). La reconstrucción y la clasificación interna de la rama kampa de la familia arawak. Ponencia presentada en el CILLA V.

Michael, L., Beier, C. y Sullón Acosta, K. (2006). Diccionario Bilingüe Iquito-Castellano, Castellano-Iquito. Recuperado de http://www.cabeceras.org/MB\&S_2006_iquito-castellano_diccionario.pdf

Mihas, E. (Ed.) (2014). Diccionario temático ilustrado del Alto Perené ashéninka-castellano. Milwaukee, Estados Unidos: Clark Graphics.

MINEDU. (2013). Documento nacional de lenguas originarias del Perú. Lima, Perú: Ministerio de Educación del Perú.

Nies, J. (Recop.) (2008). Diccionario Piro (Yine). Tokanchi gikshijikowaka-steno. Lima, Perú: ILV.

Payne, D. (1980). Diccionario ashéninca-castellano. Yarinacocha, Perú: ILV.

Payne, D. (1991). A classification of Maipuran (Arawakan) languages based on shared lexical retentions. En D. C. Derbyshire, y G. K. Pullum (Eds.), Handbook of Amazonian Languages Vol. IV (pp. 355-499). Berlín, Alemania: Mouton de Gruyter.

Renard-Casevitz, F. M. y Pacaia, C. (2004). Yavireri inti Yayenshi igiane = El dios Yabireriy su cargado Yayenshi. Lima, Perú: IFEA.

Riviale, P. (2000). Los viajeros franceses en busca del Perú antiguo (1821-1914). Lima, Perú: IFEA.

Sala, G. (1905). Diccionario, Gramática y Catecismo Castellano, Inga, Amueixa y Campa. Lima, Perú: Imprenta Nacional de Federico Barrionuevo. 
Shaver, H. (1996). Diccionario Nomatsiguenga-Castellano, Castellano-Nomatsiguenga. Lima, Perú: ILV.

Snell, B. (2008). Pequeño diccionario Machiguenga-Castellano. Lima, Perú: ILV.

Touchaux, M. (1909). Apuntes sobre la gramática y el diccionario del idioma campa o lengua de los antis tal como se usa en el río Apurimac. Lima, Perú: Imprenta Nacional de Federico Barrionuevo.

Touchaux, M. (1910). Curso práctico de quechua (dialectos del norte). Lima, Perú: Imprenta Nacional de Federico Barrionuevo.

Varese, S. (2006). La sal de los cerros: Resistencia y utopía en la Amazonía peruana. Lima, Perú: Fondo Editorial del Congreso del Perú.

Wise, J. (2017). Marcoy's Journey Across South America [entrada de blog]. Recuperado de https://www.southamerica totheworld.com/marcoys-journey-across-south-america/

Zavala, M. (1895). Vocabulario de la lengua campa. En J. Capelo, La vía central del Perú (pp. 155-164). Lima, Perú: Imprenta Masías.

\section{Notas}

1 Agradezcco a los revisores anónimos y a los editores de la revista por sus valiosas observaciones en la forma y contenido de este trabajo.

2 En esta figura, falta subdividir el ashéninka en ashéninka (sin más) y ashéninka del Pajonal.

3 Las ubicaciones de la Figura 2 (codificadas con las primeras letras del nombre de cada material) fueron provistas por ArcGis Online (https://www.esri.com/en-us/arcgis/products/arcgis-online/overview) a partir de la introducción de la información geográfica ofrecida en las fuentes.

4 Para el presente estudio, enfocado en fricativas y africadas, los lexicones fueron construidos recogiendo los lemas de los materiales que correspondieran a lexemas nominales con fonemas fricativos y/o africados en fuentes modernas de ashéninka (Payne, 1980; Mihas, 2014), asháninka (Kindberg, 1980), matsigenka (Snell, 2008) o nomatsigenga (Shaver, 1996), más otros lemas de vocabulario básico. En relación con Castelnau, Marcoy y Carrasco, se recogió exhaustivamente todos los lemas que cumplieran la condición indicada; pero en relación con los demás materiales, solo se recogió con exhaustividad los lemas que correspondieran a lemas con fricativas o africadas ante vocal /e/ o /i/, además de /9/ en nomatsigenga y / ii/ en matsigenka, mientras que los demás lemas fueron recogidos únicamente en número suficiente para conformar una muestra. Por ello, si en las tablas el número de muestras de fricativas ante /i/ es mayor que ante / a/ -por ejemplo-, de esto no debe entenderse que la primera vocal tiene un mayor rendimiento que la segunda en el material en cuestión.

5 En las Tablas 1-4 y 7-14, el número fuera de paréntesis señala la cantidad de lemas diferentes en los que aparece la grafía en cuestión, mientras que la información entre paréntesis añade la cantidad de veces en la que uno de esos lemas es transcrito alternativamente con otra grafía y cuál es esta. Así, en la Tabla 1, para la grafía <çh> en el contexto _<e,i> (esto es, que la vocal representada es /e/, pero que esta puede aparecer transcrita también como $<\mathrm{i}>$ ),debe leerse que el dígrafo aparece como tal en 4 lemas diferentes y que uno de estos exhibe 1 caso de escritura alternativa con $<$ tz $>$ en lugar $\mathrm{de}<\mathrm{çh}>$. Junto con las grafías consonánticas, entre paréntesis angulares se registra la aparición excepcional de una grafía o secuencia de grafías vocálicas distinta a la esperada.

6 Señala el autor del "arte": "Mas prevengo que en las letras $S, D, L, F$, nunca las pronuncian naturalmente como hacen todas las mas de el alfabeto [...]; porque para decir "Dios" dicen Tios, y aun la $S$ final nunca la pronuncian limpia, y dicen Tioch, haciendo en los substantivos de nombre pronombre \& de la $S$, Çhi [...]. Y así la $s$ la hacen çhi pospuesta ó antepuesta [a las vocales $<\mathrm{e}>\mathrm{o}<\mathrm{i}>$ ], pero no se entienda que la converten en $c h i$ como el español usa, si, çhi como el francés. Sirva de exemplo: este llama al caballo "cheval”; y el español "caballo" pronunciando tan suavemente la $c h$ el francés que solo se percibe... $h$ no obstante que le antecede la $c$ ” (Adam, 1890, pp. 17-18).

7 Dice el autor del "arte": "Pero la $S$ que se antepone á los substantivos y verbos, algunas veces y en algunos casos raros la pronuncian limpia, aunque la hieren mas que nosotros, v. g. en el verbo de ellos irregular "vamos” dicen tzame, y así lo escribiera mas bien con $Z$ que con $S$, “vamos todos" tzamegeito, "Simon” Tzimon. En los verbos que traen alguna $S$ intermedia, v. g. el verbo inquitzatia "enojarse”, la pronuncian líquidamente v. g. inquisatia” (Adam, 1890, p. 18). En la cita de la nota anterior y el inicio de esta, los fonos $[\mathrm{s}]$ y $\left[\int\right]$ eran "limpio" y "no limpio", respectivamente. Ahora, el valor de $\langle\mathrm{tz}>\mathrm{y}<\mathrm{z}>$ es calificado como "limpio", a la manera de [s], pero más "herido". Esto conduce hacia un tercer sonido, de carácter sibilante, pero no estridente, que puede ser confundido con [s] por el lector castellano, al que está dirigido originalmente el material, y que la comparación con las variedades campa modernas termina por identificar como [ts]. 
8 En Castelnau, se halló un solo caso de $<$ sh $>$ ante /i/ en el lema < shiyana >, glosado como 'mujer'. Este caso, sin embargo, no fue contabilizado en la Tabla 3, porque el lema no tiene correlato en las lenguas campa modernas. Además, en la entrada < pitipaxani> 'cinco' se halló un caso aparente de fricativa en la grafía $<x>$, pero la comparación con los lemas <pitipáyeni> y /pitipageni/, ambos 'cinco', de Aza Martínez de Vega (2009) y Snell (2008), respectivamente, indican que dicha grafía consistiría, más bien, en una errata por <y $>$. En Marcoy (1869), asimismo, se halló un solo caso de <ss $>$ ante /i/ en la palabra <issiti> 'sol', pero este no fue contabilizado en la Tabla 3, porque el lema en cuestión tampoco tiene correlato en las lenguas campa modernas. Finalmente, en el reporte presentado por Carrasco a propósito de la expedición, no en el material léxico analizado aquí, el autor describe a un animal "cuya figura es enteramente igual á la del zorro que se conoce en la costa" y al que, según él, los “campas" llaman "capizo" (Carrasco, 1879, p. 165), nombre que evidentemente corresponde a / kape $\int \mathrm{i} /$ 'achuni' (Nasua nasua) en ashéninka, asháninka y matsigenka y cuya grafía $<\mathrm{z}>$ parece representar un sonido diferente de $[\mathrm{s}]$, probablemente $\left[\int\right]$. Con todo, obsérvese lo excepcionales que resultan, en los materiales, estos posibles casos de grafías que representarían a un fono distinto de [s].

9 En las Tablas 5 y 6, solo se ha anotado entre paréntesis el número de muestras de las grafías menos frecuentes. En relación con las grafías que aparecen en posición de coda silábica, la letra encorchetada indica la naturaleza de la vocal precedente (núcleo de la sílaba), que no aplica para las africadas, pues estas no aparecen en coda en las lenguas pano.

10 Ver nota siguiente.

11 Castelnau (1851) ofrece también un léxico de la lengua iquito. La comparación de este material con el diccionario iquito de Michael, Beier, Sullón Acosta (2006) corrobora plenamente la conclusión de que Castelnau, o quien haya sido el transcriptor, diferenciaba entre una fricativa alveolar [s] y una alveopalatal [ $\int$ ], que representaba mediante $\langle$ s $>\mathrm{y}\langle\mathrm{sh}\rangle$, respectivamente, en su léxico iquito (léase con cuidado las pautas de escritura que aparecen en Beier (2017), donde es explícito que, en el diccionario de esta lengua, la secuencia de grafías $<\mathrm{si}>$ corresponde fonéticamente a $\left[\int \mathrm{i}\right]$ ).

12 Igual que Carrasco, Marcoy anota "sipibos” (Marcoy, 1869, p. 723), lo que invita a reconsiderar la reconstrucción de la raíz en cuestión, que Loos (2008) estableciera como */ Sipi/ 'esp. de mono' para el protopano, pero que estos datos, si son correctos, sugieren reconstruir en la forma*/sipi/, a la manera del matsés actual (ver Fleck Zuazo, Uaquí Bëso, Jiménez Huamán, 2012).

13 El autor equipara la fonética de $<$ sh $>$ su con la de la grafía $<$ sh $>$ del inglés en la palabra <shilling $>$ (Delgado, 1896-1897, p. 446)

14 Sala (1905) también ofrece un léxico yánesha. La comparación de las grafías de ese léxico con los fonemas del diccionario yánesha de Martha Duff-Tripp (Duff-Tripp, 1998) corrobora los valores fonéticos atribuidos aquí a las grafías del material campa de Sala.

15 Dice Marcelo Touchaux: "sh se pronuncia como en el quechua de Huaraz; shimi: palabra,-ó como la ch francesa, vg.: chien" (Touchaux, 1909, p. 8); esto es, como un fono [ $\left.\int\right]$. El autor también escribió una gramática de quechua central (Touchaux, 1910), cuya revisión y comparación con gramáticas modernas de quechua central corrobora que el autor distinguía de manera inequívoca entre los fonos $[\mathrm{s}] \mathrm{y}\left[\mathrm{\int}\right]$, así como entre $[\mathrm{t}]$ y $[\mathrm{ts}]$, y que representaba el segundo término de cada par mediante las grafías $<\mathrm{sh}>\mathrm{y}<\mathrm{ts}>$, respectivamente.

Dice José Pío Aza Martínez de Vega: “Como el machiguenga carezca de escritura, para la parte gráfica me he servido del alfabeto español. Pero hay dos sonidos a los cuales no corresponde signo alguno de nuestro alfabeto: el uno es igual al que expresan los ingleses con la sh, los franceses con $c h$, y los alemanes con la sch. Entre estos he preferido la forma inglesa porque se presta a menor confusión" (Aza Martínez de Vega, 2009 [1924], p. 257). Nuevamente, el fono aludido es [S].

16 En Aza Martínez de Vega (2009 [1924]), la <ch>también es descrita como correspondiente a [ $\mathrm{t}$ ]. Véase las últimas frases de la cita en la nota siguiente.

17 La cita de Aza Martínez de Vega de la nota 15 continúa así: "Para expresar el otro sonido me valgo de la $c h$, por tener alguna similitud con el de esta letra: pero para que no se confunda y se advierta que su pronunciación es distinta de la $c h$ castellana, llevará como signo diacrítico estar presentada por diferente tipo de letra del resto del vocablo, v. g. iramburiCHa, eiCHa, nobaCHa. La $c h$ con su sonido idéntico al del castellano es muy frecuente en el machiguenga, y así se dice chichi, pangochi, kibaitankichane, etc." (Aza Martínez de Vega, 2009 [1924], p. 257). Sin embargo, en toda la extensión del material, el diacrítico utilizado por el autor para la representación del "otro sonido" parecido a $<\mathrm{ch}>$ no son las letras altas, sino las cursivas. En el presente trabajo, la grafía en cuestión es presentada en altas para que se distinga claramente de $<\mathrm{ch}>$.

18 Obsérvese muy especialmente en (Aza Martínez de Vega, 2009 [1924]) que, si bien el fono [ts] puede ser representado mediante $\langle\mathrm{s}\rangle,\langle\mathrm{ts}\rangle,\langle\mathrm{tz}\rangle \mathrm{o}\langle\mathrm{CH}\rangle$, ninguno de estos recursos gráficos aparece ante la vocal /i/, lo que refuerza la conclusión de que no había /ts/ ante /i/ en la variedad en cuestión.

19 En el léxico campa, dos ejemplos claros de la particularidad de cada material ocurren alrededor de las palabras para 'tabaco' y 'puerco salvaje', a las que Castelnau responde con <seri $>$ y $<$ sintori $>$; Marcoy, con < sairi $>$ y $<$ sintuli $>$; y Carrasco con $<$ siri $>$ y la palabra $<$ quintasiri $>$, que no tiene correlato entre las variedades actuales. Asimismo, en el léxico pano, a la palabra para 'diablo', Castelnau responde con $<$ yaunchi $>$; Marcoy, con $<$ yurima $>(<\mathrm{r}>$ se correlaciona con 
la $<$ ch $>$ de los otros, ver Tabla 5); y Carrasco, con $<$ illuchi $>$, donde la correlación de $<$ ll $>$ con la $<y>$ de los franceses evidencia el "yeísmo" del autor peruano. No obstante, no se puede dejar de hacer énfasis en las "anomalías" en común entre Marcoy y Carrasco respecto del léxico campa, como la transcripción <imchato $>$, con $<$ m $>$ en lugar de $<$ n $>$, para el lexema /int fato/ 'arbol', o los respectivos lemas <maguani $>$ y <muguani >, ambos 'cuatro', cuando en Castelnau y en el resto de lenguas campa modernas la raíz / maßa/ significa, más bien, 'tres'.

20 Recuérdese lo señalado en la nota 11.

21 Cotéjese los cognados <genoqui ' 'arriba', <peronti> 'luciérnaga' y <mape, mapi> 'piedra' en De la Marca; $<$ mapui> en Castelnau; <mapi> en Marcoy; <mapi> en Carrasco; <uniqui>, <pirontsi> y <mappi> en Zavala; <perontzi> y <mapí $>$ [alomorfo singular] <mapié $>$ [alomorfo plural] en Delgado (ver nota 22); <enoqui, iniqui, noqu $i>$, $<$ pironchi> y <mappi> en Sala; <enoque> y <mapi> en Touchaux; <enokui $>$, <puiro> y <mapue> en Aza (ver */ henokti/ 'arriba' y*/mapti/ 'piedra' en protoarahuaco [Payne, 1991]).

22 Cotéjese los cognados <quitaiteri> 'día, amanecer' y <camaqui > diablo' en De la Marca; kita[e]teri en Castelnau; <quitahuiti> y <camacarinchi> en Marcoy; <quitaguititai> y <camacarinchi> en Carrasco; <quitéiteri > y <camali> en Zavala; <quitaiteri> en Delgado; <quiteiti, quitaitiri> y < camari> en Sala; <quitaguiti> y <camagari> en Touchaux; $<$ kuitaguiteri> y < camagarine> en Aza (cf. */ktitagiti/ 'día' en protoarahuaco [Payne, 1991]).

23 La comparación de los léxicos "pano", "conibo" y "sipibo" de Castelnau, Marcoy y Carrasco con los léxicos modernos de kapanawa (Loos y Loos, 2003) y shipibo (Loriot et al., 1993), así como la de los léxicos de "piro chontaquiro" de Castelnau y Marcoy con el léxico yine (piro) moderno de Nies (2008), indica que los tres autores del siglo XIX estaban en capacidad de registrar y anotar de manera sistemática e inequívoca (como una secuencia vocálica $\langle$ ue $\rangle$ u $\langle$ oe $\rangle$ ) una vocal central $/ \mathbf{t} /$ solo cuando esta era precedida por una consonante labial. En sus respectivos léxicos campas, esta condición ocurre únicamente en la palabra para 'piedra' (protocampa */mapti/), que Castelnau, Marcoy y Carrasco transcribieran como <mapui $>$, <mapi $>$ y $<$ mapi $>$, como fue señalado en nota anterior. Este hecho conduce aquí a clasificar el primer material como correspondiente a una variedad de la rama NMN, que no experimentó el cambio */ti/ $>$ /i/ (de ahí, el diptongo <ui>), mientras que los otros dos, a una de la rama KAA, que sí lo experimentó (de ahí, la transcripción $<\mathrm{i}>$ ). También la comparación de Aza con el léxico matsigenka de Snell (2008) revela, en Aza, el registro del diptongo $/ \mathrm{i} \mathrm{i} /$ como $<\mathrm{u}>$ o un diptongo que comienza con esta letra -aunque hay excepciones-; por eso, también este material es clasificado en la rama NMN. Fuera de esto, Delgado (1896-1897) registra un único caso de diptongo <ei> precisamente en una muestra de la raíz para 'piedra' cuando esta recibe el sufijo de plural /-paje/: <mapíe-pai> 'piedras', como también quedó señalado en nota anterior. Frente a las numerosas muestras de $<\mathrm{i}>$ en Delgado que corresponden a / $\mathrm{t}$ / en matsigenka, dicho caso aislado es considerado aquí una preservación del diptongo protocampa en el alomorfo de la raíz en cuestión, si es que no se trata de una errata, y el material es, consecuentemente, clasificado como KAA junto con el resto de materiales, que no son problemáticos en este punto. 